\title{
Eclipsing binaries in the Magellanic Clouds ${ }^{\star}$
}

\section{uvby CCD light curves and photometric analyses for HV 982 (LMC), HV 12578 (LMC), HV 1433 (SMC), and HV 11284 (SMC) ${ }^{\star \star}$}

\author{
J. V. Clausen ${ }^{1}$, J. Storm ${ }^{2}$, S. S. Larsen ${ }^{1,3}$, and A. Giménez ${ }^{4,5}$ \\ ${ }^{1}$ Niels Bohr Institute for Astronomy, Physics and Geophysics, Astronomical Observatory, Juliane Maries Vej 30, \\ 2100 Copenhagen $\varnothing$, Denmark \\ e-mail: jvc@astro.ku.dk \\ 2 Astrophysikalisches Institut Potsdam, An der Sternwarte 16, 14482 Potsdam, Germany \\ e-mail: jstorm@aip.de \\ 3 UC Observatories/Lick Observatory, University of California, Santa Cruz, CA 95064, USA \\ e-mail: soeren@ucolick.org \\ ${ }^{4}$ Laboratorio de Astrofísica Espacial y Física Fundamental INTA, Estacion de Villafranca del Castillo, Apdo. 50727, \\ 28080 Madrid, Spain \\ 5 Research and Scientific Support Department, ESA, ESTEC, Postbus 299, 2200 AG Noordwijk, The Netherlands \\ e-mail: agimenez@rssd.esa.int
}

\section{Received 30 August 2002 / Accepted 16 December 2002}

\begin{abstract}
We present new accurate CCD uvby light curves for the LMC eclipsing binaries HV 982 and HV 12578, and for the SMC systems HV 1433 and HV 11284 obtained at the Danish $1.5 \mathrm{~m}$ telescope at ESO, La Silla. The light curves were derived from DoPHOT photometry, and typical accuracies are between 0.007 and 0.012 mag per point. Standard $u v b y$ indices have also been established for each binary, primarily for determination of interstellar reddening and absorption. For HV 982 and HV 12578, accurate photometric elements have been established. Both systems consist of two detached components of comparable sizes in an eccentric orbit. Adopting the spectroscopic elements given by Fitzpatrick et al. (2002) for HV 982, we derive absolute dimensions of its components which agree well with their results. A distance modulus of $V_{0}-M_{V}=18.63 \pm 0.08$ is obtained, corresponding to a distance of $53.2 \pm 2.0 \mathrm{kpc}$, which is in formal agreement with (although slightly larger than) their determination. HV 1433 and HV 11284 both consist of two rather close, deformed and quite different stars. As the mass ratios between the components (and their rotation rates) are not known, definitive photometric elements can not yet be obtained, but we present a sample of possible photometric solutions. In a series of forthcoming papers we will combine our $u v b y$ observations with high-dispersion spectra from the UVES spectrograph on the ESO Very Large Telescope (VLT) and present absolute dimensions, chemical abundances and distances for selected LMC and SMC systems, including HV 12578 and refined results for HV 982.
\end{abstract}

Key words. cosmology: distance scale - galaxies: magellanic clouds - stars: fundamental parameters - stars: binaries: close stars: binaries: eclipsing - techniques: photometric

\section{Introduction}

Double-lined eclipsing binaries provide, apart from objects where purely geometrical methods can be applied, the most

Send offprint requests to: J. V. Clausen,

e-mail: jvc@astro.ku.dk

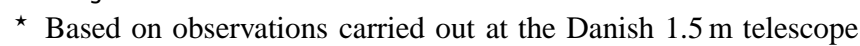
at ESO, La Silla, Chile.

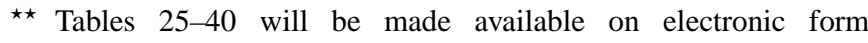
at the CDS via anonymous ftp to cdsarc.u-strasbg.fr (130.79.128.5) or via

http://cdsweb.u-strasbg.fr/cgi-bin/qcat?]/A+A/402/509 direct distance determinations available. From complete, accurate observations and careful analyses of well-detached systems, distance moduli better than \pm 0.10 mag can be obtained.

With present-day facilities, including ground based 10-m class telescopes and the Hubble Space Telescope (HST), precise studies of systems in Local Group galaxies, primarily the Magellanic Clouds (MC), have become possible. Therefore, accurate LMC and SMC distances can now be established from eclipsing binaries, leading also to the calibrations needed for other important extragalactic distance indicators, like e.g. Cepheids, reaching far beyond the Local Group. Reviews on 
the subject have recently been presented by Paczýnski (1997) and Clausen (2000).

Also, the accurate absolute dimensions obtained for the MC binary components makes it possible to gain insight into e.g. core structure (e.g Ribas et al. 2000) and mass loss for massive stars through comparison with stellar models. Furthermore, they provide fundamental mass-to-luminosity (M/L) relations for metal-deficient stars of e.g. typical LMC/SMC compositions.

The first accurate CCD light curves of LMC and SMC eclipsing binaries were obtained at the Danish $1.5 \mathrm{~m}$ telescope at ESO, La Silla by Jensen et al. (1988), who give references to earlier photometry. The present paper should be seen as a continuation of this work. Meanwhile, dedicated CCD light curve observations have been done for several LMC and SMC systems at Mt. John University Observatory, New Zealand (Pritchard et al. 1998a, 1998b and references therein), and several thousands of new systems have been discovered from the EROS, MACHO, and OGLE microlensing projects (e.g. Grison et al. 1995; Alcock et al. 1997; Udalski et al. 1998b).

Low to medium resolution spectroscopy is available for a few MC systems (e.g. Niemela \& Bassino 1994), and moderately accurate distances and dimensions based on CCD light curves and radial velocities from such spectroscopy have been published for some systems, e.g. HV 2241 (Pritchard et al. 1998a), HV 2543 (Ostrov et al. 2000), and HV 5936 (Bell et al. 1993) in the LMC, and HV 1620 (Pritchard et al. 1998a) and HV 2226 (Bell et al. 1991) in the SMC.

However, an important step forward was recently taken when a very accurate distance modulus (about $\pm 0.07 \mathrm{mag}$ ) and stellar dimensions (about $\pm 2.5 \%$ for radii and $\pm 6 \%$ for masses) were determined for the LMC system HV 2274 (Watson et al. 1992; Guinan et al. 1998; Udalski et al. 1998a; Nelson et al. 2000; Ribas et al. 2000; Groenewegen \& Salaris 2001). Masses and radii were obtained from normal analyses of light curves and radial velocity curves, based on rather few high dispersion spectra, whereas effective temperatures, reddenings and distances were obtained from UV/optical spectrophotometry. Slightly deviating distances are obtained from the different studies, mainly due to differences in the reddening determinations, but they all tend to support the short LMC distance scale (e.g. Gibson 2000).

Very recently, accurate distances and dimensions for two additional LMC systems, HV 982 (Pritchard et al. 1998b; Fitzpatrick et al. 2002) and EROS1044 (Ribas et al. 2002), have become available. A comparison of the results for HV 2274, HV 982, and EROS1044, and a discussion of the derived distances to the optical center of the LMC is given by Ribas et al. (2002). Clearly, studies of many more binaries, well distributed across the LMC, are needed, and are important also for investigations on the orientation and structure of the galaxy. SMC systems should also be included to extend the investigation to significantly lower metallicities, which will also allow a study of the metallicity dependence of the Cepheid period-luminosity relation.

In this paper, we present new complete uvby light curves and analyses of them for the LMC systems HV 982 and HV 12578, and the SMC system HV 11284, and partial
Table 1. The observed eclipsing binaries. For comparison, the position of the optical centers of LMC and SMC are included.

\begin{tabular}{lllll}
\hline \hline ID & $V_{\max }$ & $P_{\text {days }}$ & $\alpha(2000.0)$ & $\delta(2000.0)$ \\
\hline LMC & & & $05^{\mathrm{h}} 24^{\mathrm{m}}$ & $-69^{\circ} 45^{\prime}$ \\
HV 982 & 14.7 & 5.34 & $05^{\mathrm{h}} 29^{\mathrm{m}} 53^{\mathrm{s}}$ & $-69^{\circ} 09^{\prime} 23^{\prime \prime}$ \\
HV 12578 & 15.8 & 2.48 & $05^{\mathrm{h}} 21^{\mathrm{m}} 32^{\mathrm{s}}$ & $-66^{\circ} 21^{\prime} 15^{\prime \prime}$ \\
SMC & & & $00^{\mathrm{h}} 53^{\mathrm{m}}$ & $-72^{\circ} 50^{\prime}$ \\
HV 1433 & 16.4 & 2.05 & $00^{\mathrm{h}} 47^{\mathrm{m}} 11^{\mathrm{s}}$ & $-73^{\circ} 41^{\prime} 18^{\prime \prime}$ \\
HV 11284 & 17.1 & 3.63 & $00^{\mathrm{h}} 49^{\mathrm{m}} 43^{\mathrm{s}}$ & $-72^{\circ} 51^{\prime} 10^{\prime \prime}$ \\
\hline
\end{tabular}

results for the SMC system HV 1433. Studies of reddening and metallicity in the observed fields (about $6.5 \times 6.5 \mathrm{arcmin}$ ) centered on the four systems have previously been published (Larsen 1996; Larsen et al. 2001).

Further, together with a group of colleagues we are undertaking a large scale project, combining light curve observations, standard photometry, and high dispersion spectroscopy from the ESO VLT UT2 telescope and the new very efficient UVES echelle spectrograph (Dekker et al. 2000; D'Odorico 2000). Until now, complete spectroscopic observations have been obtained for four LMC systems, including HV 982 and HV 12578, and two SMC systems, and analyses are in progress.

\section{Selection of candidates}

When the $u v b y$ observations were planned, the thousands of newly discovered MC binaries from the microlensing projects were not yet available, and our source for the selection was then limited to the about 110 systems identified mainly from the photographic material of the Harvard surveys (Shapley \& Nail 1942, 1953; Payne-Gaposchkin \& Gaposhkin 1966; Gaposhkin 1970, 1977; Hodge \& Wright 1967, 1977; Kreiner 1972).

About 10 candidates per galaxy were selected, but for several reasons (field crowding, faintness, difficult orbital periods, lack of observing time, etc.) the project was gradually concentrated on the four systems listed in Table 1. Part of the fields, centered on the eclipsing binaries, are shown in Figs. 14.

HV 982 is located about one degree NE of the optical center of LMC, whereas HV 12578 is located in the northern outskirts of the LMC Bar, about three degrees from the optical center. HV 1433 is located in the southern part of the central region of SMC, just south of the OGLE - II field SMC_SC4 (Udalski et al. 1998b), and HV 11284 slightly west of the optical center of SMC in the OGLE - II field SMC_SC5. HV 11284 is identical to the OGLE - II eclipsing binary SMC_SC5_140701.

\section{Observations and basic reductions}

The CCD observations were carried out during several periods between November 1992 and November 1995 at the Danish $1.54 \mathrm{~m}$ telescope at ESO, La Silla, equipped with a direct camera and CCD \#28 (a thinned $1024 \times 1024$ Tek device). This combination of telescope and CCD yields a field size of about $6.5 \times 6.5 \mathrm{arcmin}$. The image scale is $0.38^{\prime \prime} /$ pixel. The 


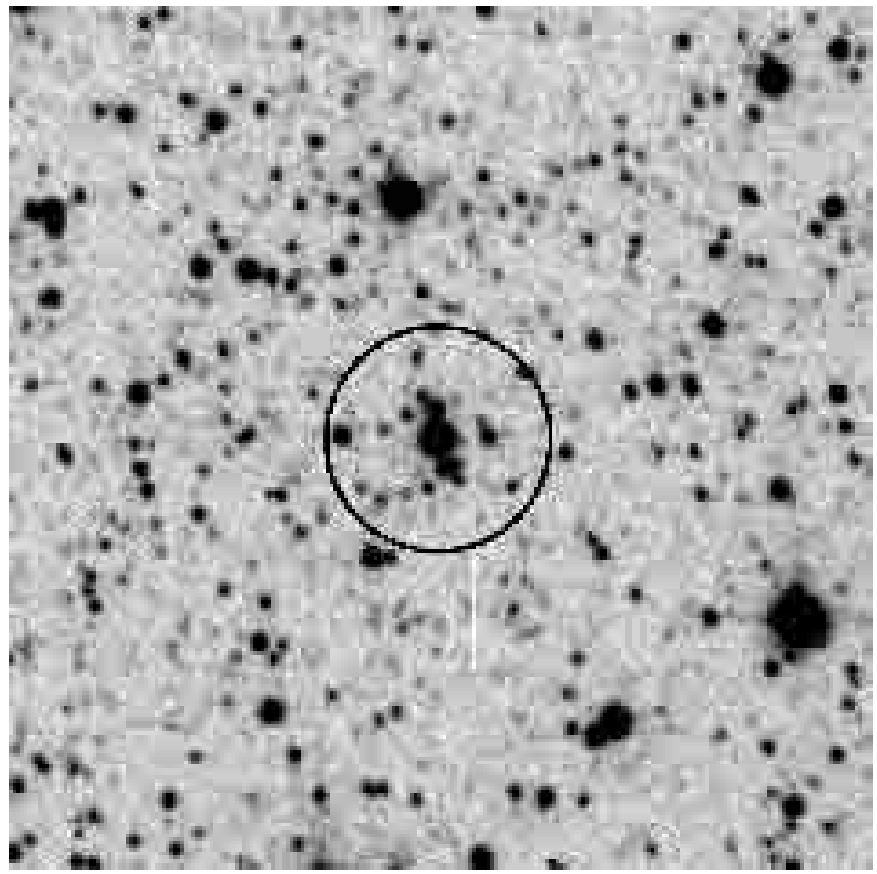

Fig. 1. HV 982 and the surrounding field (1.5 arcmin square). The $y$ image was observed at 1.0 arcsec seeing. North is up, and east is to the left.

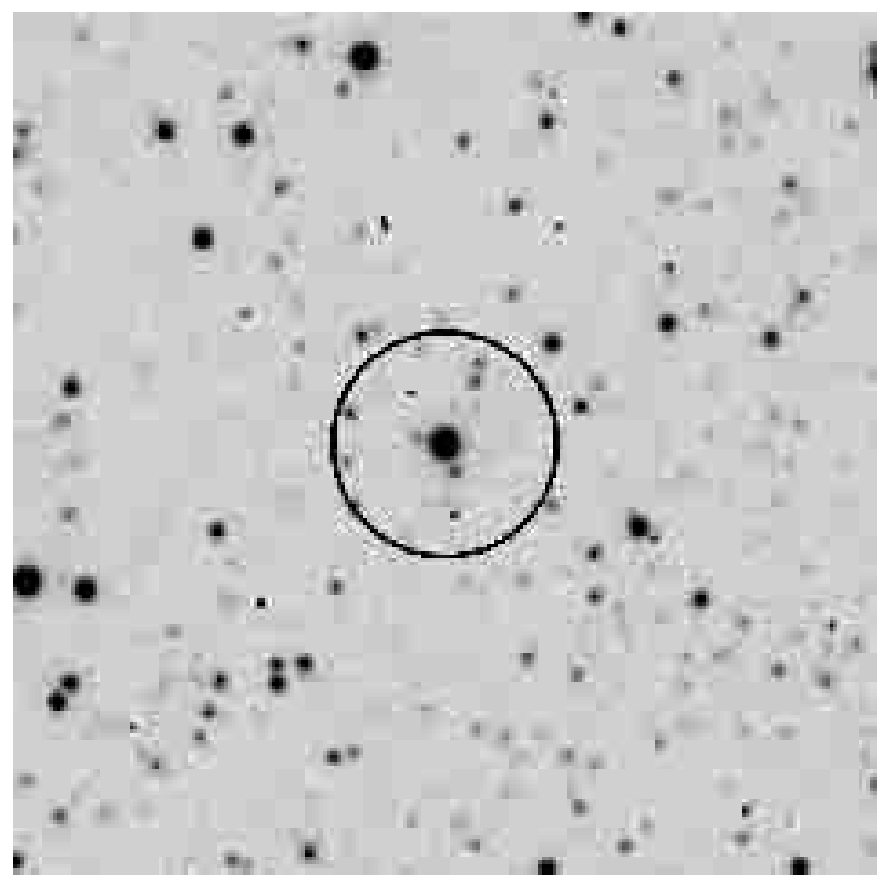

Fig. 2. HV 12578 and the surrounding field (1.5 arcmin square). The $b$ image was observed at 1.1 arcsec seeing. North is up, and east is to the left.

same set of uvby interference filters were used throughout, except for the early $1992 b$ observations. Photometry from several $b$ images of the same field obtained with the two filters were carefully compared, but no significant differences between the instrumental systems were found.

The binaries were always positioned near the field centers, but no effort was made to place them on exactly the

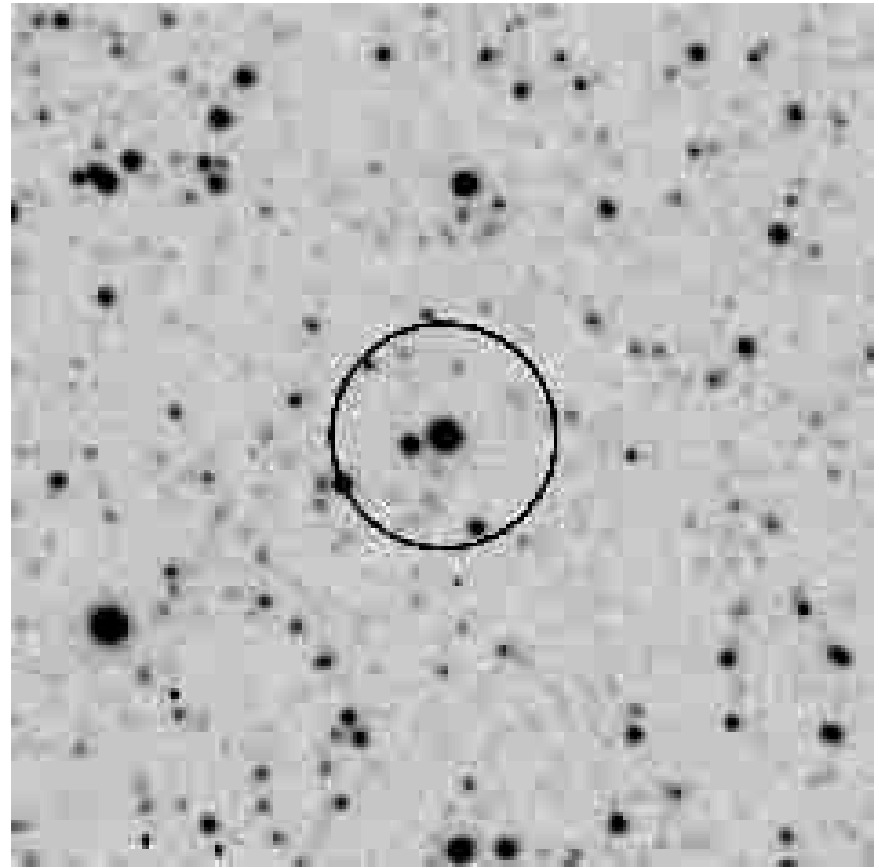

Fig. 3. HV 1433 and the surrounding field (1.5 arcmin square). The $y$ image was observed at 1.1 arcsec seeing. North is up, and east is to the left.

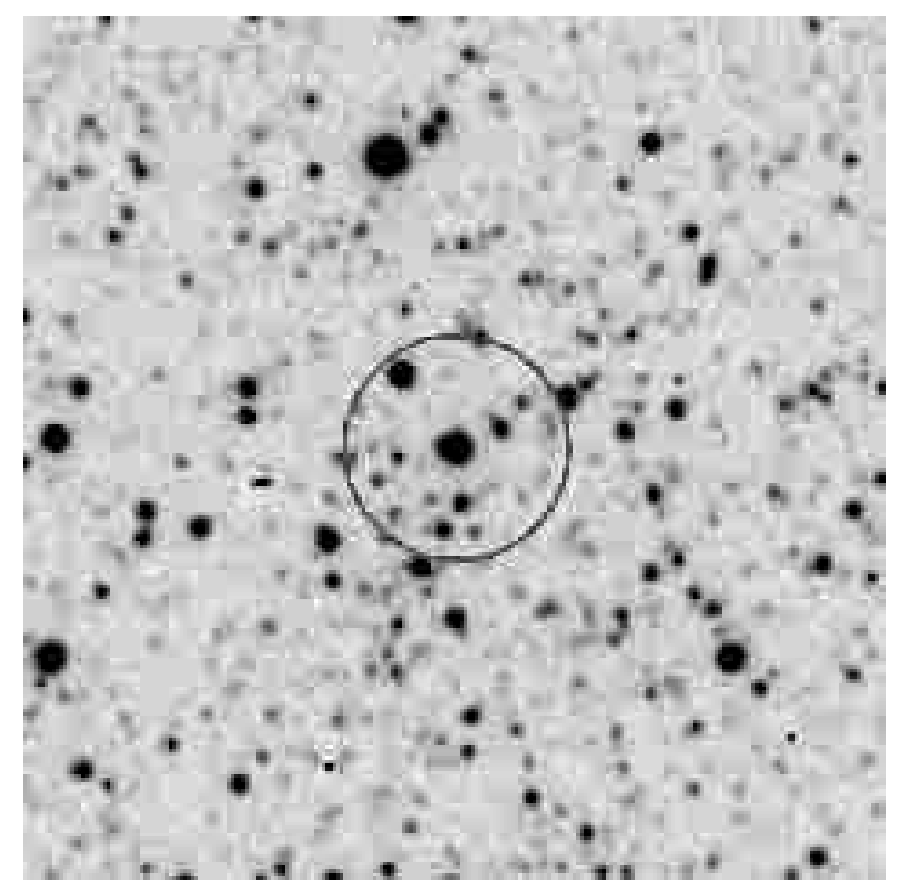

Fig. 4. HV 11284 and the surrounding field (1.5 arcmin square). The $b$ image was observed at 1.1 arcsec seeing. North is up, and east is to the left.

same pixels on all exposures. Also, the orientation of the camera/chip varied between the different observing runs. Typical exposure times for HV 12578, HV 11284, and HV 1433 were 5 (vby) and $20 \mathrm{~min}(u)$, but for the brighter HV 982 we used 2.5 and $10 \mathrm{~min}$, respectively. The observations were in general done in ybvyu sequences in order to obtain complete 
light curves in one band $(y)$ and acceptable coverage in the other bands.

Initial reductions, essentially dark current and bias subtractions and flatfielding, where carried out using standard IRAF ${ }^{1}$ tools. As the dark current was very low, only around 4 counts per hour with no "hot spots" or other defects visible, we decided to use just a single common value for the dark current in order not to introduce additional noise. In each observing run, typically 10 bias exposures were made, and in all cases the bias level was found to be constant within 1-2 ADU during a given run, whereas the level changed slightly from year to year, depending on the electronic setup of the CCD. The bias frames were all very uniform, the only defect being a weak pattern from the thinning of the chip, and we used combined frames for the bias subtraction. In each observing run, sky flatfield exposures were made every evening and morning, and the individual frames, typically 5 per filter with slight offsets between them, were carefully checked against a reference frame obtained with the same filter. Any exposures that contained bright stars or other obvious anomalies were rejected. For the remaining exposures, we found no obvious variations from night to night or between morning and evening flats, and we thus decided to use a single combined flatfield in each filter for each run. The accepted flatfield exposures were combined applying a sigmaclipping rejection algorithm to avoid any (faint) stars that might be present in the images.

As a last step, we measured the seeing from two or more stars in each frame, and determined the sky background level. This information is given in Tables 25-40 (light curve tables, instrumental system). No images with seeing above about 3 arcsec were accepted, and most images were obtained at 1-1.5 arcsec seeing. Very few images with a background above $100 \mathrm{ADU}$ were accepted.

Additional information on the reduction is given by Larsen (1996).

\section{Photometry and formation of light curves}

\subsection{Light curves}

Several packages are available for crowded field photometry, including MOMF (Kjeldsen \& Frandsen 1992), ROMAPHOT (Buonnano et al. 1993), DoPHOT (Schechter et al. 1993), and DAOPHOT (Stetson 1987). Especially the latter two have been widely used, and while DAOPHOT may be the most flexible of the two, DoPHOT is particularly well suited for reductions of large quantities of data because only a minimum of user interaction is required. Tests of DoPHOT and DAOPHOT by ourselves and others (e.g. Larsen 1996; Ferrarese et al. 2000) show that the two packages generally provide photometry of comparable accuracy, as well as very realistic error estimates. Because of its large number of user-definable parameters and greater flexibility, DAOPHOT may give slightly more accurate photometry in difficult cases such as strongly crowded

\footnotetext{
${ }^{1}$ IRAF is distributed by the National Optical Astronomical Observatories, which are operated by the Association of Universities for Research in Astronomy, Inc. under contract with the National Science Foundation.
}

Table 2. Mean DoPHOT errors (mag).

\begin{tabular}{lrrrr}
\hline \hline Binary & $y$ & $b$ & $v$ & $u$ \\
\hline HV 982 & 0.008 & 0.007 & 0.008 & 0.008 \\
HV 12578 & 0.009 & 0.008 & 0.009 & 0.010 \\
HV 1433 & 0.009 & 0.008 & 0.010 & 0.012 \\
HV 11284 & 0.008 & 0.007 & 0.008 & 0.009 \\
\hline
\end{tabular}

fields, or if the PSF varies significantly across the frame. This may be partly due to the more sophisticated PSF modeling in DAOPHOT. However, DoPHOT also produces very good photometry and runs substantially faster than DAOPHOT. Because of the large volume of data to be reduced in this project, we therefore opted to use DoPHOT for the analysis of the LMC and SMC fields.

Following the DoPHOT analysis, the photometry output files were combined into a single database for each binary field using the DBMAG package developed by one of us (JS). Besides efficient book keeping and photometry extraction tools, it contains tasks for identification and detection of variable stars based on the technique by Welch \& Stetson (1993). For each binary field and photometric band, a master frame was selected, and all similar frames were then brought to the zero point of the master.

As a final check of the procedure, we studied one of the databases, the $y$ band of the HV 11284 field, in further detail. A number of isolated, supposedly constant stars spanning the magnitude range of HV 11284 through its orbit were extracted, and their mean magnitude and the corresponding rms per observation were calculated. No systematic patterns in the individual deviations from the means were seen for the stars, and rms values between 0.009 and 0.011 mag were obtained. For all stars, the rms values are close to the DoPHOT error estimates of the single magnitudes, and we conclude that the frames have been safely brought to the same zero point. Therefore, we have chosen to apply directly the database results for the binary light curves, rather than basing them on a smaller number of preselected comparison stars, as is often done.

The light curve observations in the instrumental system are presented in Tables 25-40, which will only be available in electronic form. For each light curve point, the internal frame number, the Heliocentric Julian Date corresponding to the time of mid-exposure, the orbital phase, the magnitude and its error (DoPHOT), the sky background level (in ADU), and the seeing (in pixels, the scale is $0.38^{\prime \prime} /$ pixel) are given. Typical errors per point are close to $0.01 \mathrm{mag}$ (Table 2).

Phases were calculated from the individual ephemerides given below. When possible, times of minima were calculated from the Kwee \& Van Woerden (1956) method. Ephemerides were, if possible, determined from least squares fits to new and published times of minima, else by fitting the observations together applying the method by Lafler \& Kinman (1965). The resulting light curves are shown in Figs. 6, 11, 15, and 16.

\section{Standard $u v b y$ indices}

In order to obtain reliable apparent magnitudes and colors for the eclipsing binaries, essential for reddening and distance 
determinations, a careful transformation to the uvby standard system is needed. We have based this calibration on numerous CCD observations of secondary uvby standard, for which photoelectric observations were simultaneously done at the Strömgren Automatic Telescope (SAT) at ESO, La Silla. The SAT observations also provided accurate nightly extinction coefficient needed for the calibration of the CCD photometry. The SAT observations and standard $u v b y$ indices for the secondary standards are described by Clausen et al. (1997).

Because DBMAG automatically adjusts the photometry of all individual frames to a common zero-point, it was sufficient to derive a photometric transformation to the $u v b y$ standard system for the reference frames. For this purpose, we first used the substar task in the IRAF DAOPHOT package to subtract all stars except a few bright ones from the reference frames. Aperture photometry for these stars was then obtained with the phot task, thus providing a set of "tertiary standard stars" in each frame (see Larsen et al. 2001 for details). Finally, the DoPHOT photometry was tied into the standard system by determining the offsets between the DoPHOT magnitudes and aperture photometry for the tertiary standard stars. Figure 5 shows the difference between the DoPHOT and aperture photometry for the selected tertiary standards in the HV 982 field. Typical uncertainties on the zero-points were estimated to be 0.01-0.02 mag.

We further checked the accuracy of the photometric zeropoints by comparing DAOPHOT PSF-fitting photometry on the co-added frames (Larsen et al. 2001) with the mean DoPHOT magnitudes from the DBMAG database. For the LMC fields, the comparison showed excellent agreement between the DoPHOT and DAOPHOT photometry, with systematic differences $<0.01 \mathrm{mag}$ in $y$ and $b-y$ and $<0.02 \mathrm{mag}$ in $m_{1}$ and $c_{1}$. For the SMC fields we found slightly larger differences of $\sim 0.02$ mag in $y$ and up to 0.03-0.04 mag in $m_{1}$, but still compatible with the estimated zero-point uncertainties on the photometry. Note that the zero-points were established for the individual passbands, leading to larger formal uncertainties on indices such as $m_{1}$ and $c_{1}$, which are based on three bands each.

The resulting standard $u v b y$ indices for the eclipsing binaries are given in Table 3. Intrinsic standard $u v b y$ indices, interstellar reddening, and absorption derived from the $(b-y)_{0}-c_{0}$ relation by Crawford (1978) are given in Table 4. For HV 982, the reddening determined from the uvby photometry agrees well with that recently published by Fitzpatrick et al. (2002), $E(B-V)=0.086 \pm 0.005$, based on a completely different and much more complex approach.

\section{HV 982 (LMC)}

Already the photographic Harvard Survey data (Gaposhkin 1970) show that HV 982 is a well-detached eclipsing binary, presumably with an eccentric orbit. CCD light curves obtained at Mt. John University Observatory, New Zealand by Pritchard et al. (1998b) clearly confirm these characteristics. Furthermore, with new minima observations, apsidal motion with a period of about $200 \mathrm{yr}$ could be detected, making HV 982 just the second MC eclipsing binary with measured

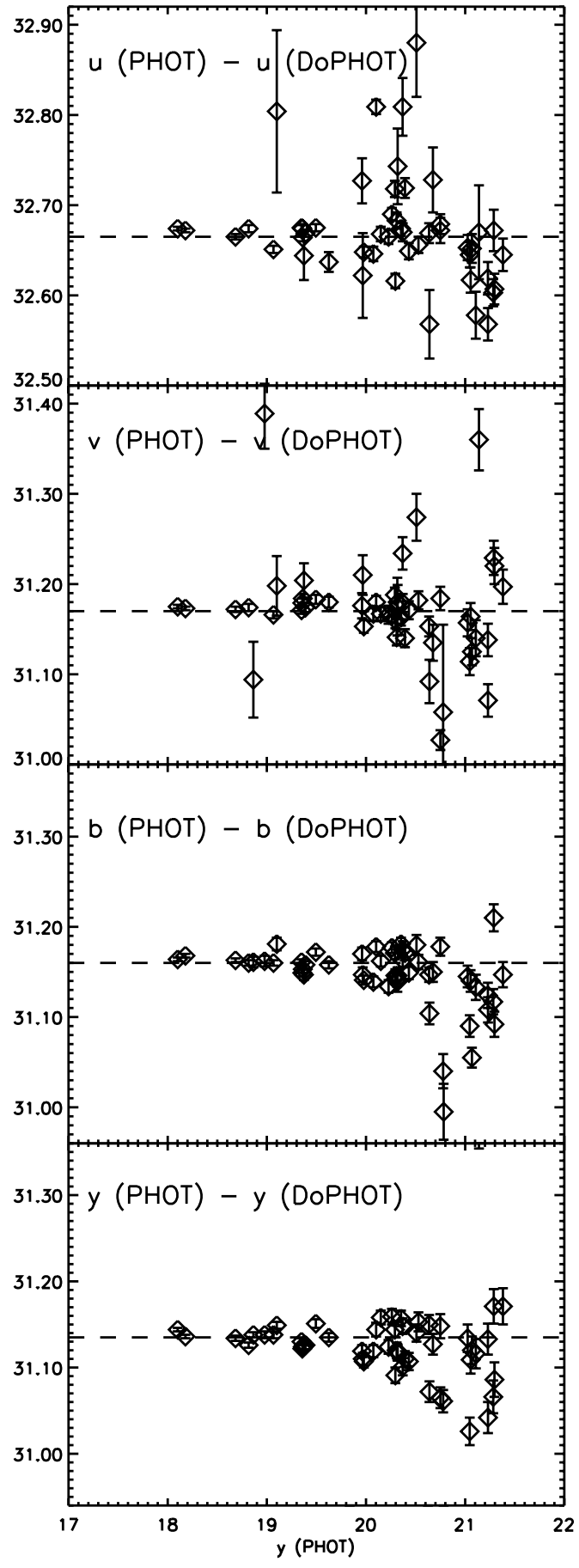

Fig. 5. Zero-point differences between aperture and DoPHOT photometry for "tertiary standards" in the HV 982 field. The dashed lines indicate the adopted zero-points.

orbital motion (the first known case is HV 2274, also in the LMC; Watson et al. 1992; Ribas et al. 2000).

Pritchard et al. (1998b) also did standard uvby photometry and IUE ultraviolet spectroscopy, and determined interstellar reddening and photometric elements for HV 982. Subsequently, Pritchard \& Niemela (2000) presented radial velocities and derived mean masses and radii for the components, and a distance modulus of $V_{0}-M_{V}=18.7 \pm 0.15$ was obtained. Very recently, Fitzpatrick et al. (2002) published 
Table 3. Standard $u v b y$ indices for the eclipsing binaries (no correction for reddening applied) at maximum light level outside eclipses. $N$ is the number of observations used to form the mean value. $\sigma$ is the rms error (per mag/index) given in mmag.

\begin{tabular}{lrrrrrrrrrrrr}
\hline \hline ID & $V$ & $\sigma$ & $N$ & $(b-y)$ & $\sigma$ & $N$ & $m_{1}$ & $\sigma$ & $N$ & $c_{1}$ & $\sigma$ & $N$ \\
\hline HV 982 & 14.648 & 8 & 69 & -0.042 & 9 & 47 & 0.070 & 15 & 44 & 0.020 & 17 & 42 \\
HV 12578 & 15.768 & 6 & 27 & -0.050 & 10 & 20 & 0.094 & 13 & 16 & 0.077 & 25 & 11 \\
HV 1433 & 15.547 & 11 & 3 & -0.070 & 5 & 4 & 0.106 & 14 & 4 & 0.014 & 25 & 3 \\
HV 11284 & 15.179 & 7 & 15 & -0.023 & 10 & 27 & 0.094 & 15 & 21 & 0.069 & 13 & 17 \\
\hline
\end{tabular}

Table 4. Intrinsic standard $u v b y$ indices at maximum light level outside eclipses, and interstellar reddening and absorption for the eclipsing binaries.

\begin{tabular}{lrrrrrrr}
\hline \hline ID & $V_{0}$ & $(b-y)_{0}$ & $m_{0}$ & $c_{0}$ & $E(b-y)$ & $E(B-V)$ & $A_{V}$ \\
\hline HV 982 & 14.350 & -0.111 & 0.091 & 0.006 & 0.069 & 0.097 & 0.298 \\
HV 12578 & 15.529 & -0.106 & 0.111 & 0.066 & 0.056 & 0.078 & 0.239 \\
HV 1433 & 15.369 & -0.111 & 0.118 & 0.006 & 0.041 & 0.057 & 0.178 \\
HV 11284 & 14.818 & -0.107 & 0.119 & 0.052 & 0.084 & 0.118 & 0.361 \\
\hline
\end{tabular}

accurate dimensions for HV 982 and found its distance modulus to be $V_{0}-M_{V}=18.50 \pm 0.06$. The results were obtained by adding HST UV/optical spectrophotometry and radial velocities based on high-resolution optical spectroscopy from the Blanco 4-m telescope at Cerro Tololo, Chile to existing photometry for HV 982.

As HV 982 is clearly an important LMC eclipsing binary, we decided - parallel to the Mt. John observations - to gain further light curve data. Mainly because the field near the system is quite complicated, with several close neighbours (Fig. 1), a delicate subtraction procedure was needed at the average Mt. John seeing of 3-5 arcsec (Pritchard et al. 1998b). This can be avoided at the average La Silla seeing of about 1-2 arcsec. Also, better $u v b y$ standard indices were needed.

In this paper, we present the light curves and photometric elements derived from them, plus standard uvby photometry. Also, absolute dimensions and distances are derived, but we expect to publish significantly improved results when the analyses of the VLT/UVES spectra has been completed.

\subsection{Light curves}

HV 982 was observed on 43 nights between JD 2448970 and JD 2450047, and 314(y),124(b), $115(v)$, and $103(u)$ points were obtained in the four bands. The light curves are given in Tables 25-28 and shown in Fig. 6. The coverage in $y, b$, and $v$ is fairly complete, whereas a gap exists in the $u$ observations of primary eclipse. Typical accuracies per point are given in Table 2.

The two eclipses are of comparable depths with central secondary eclipse occurring at phase 0.428 . No significant change of the $(b-y)$ color is seen during the eclipses. Slight reflection effects are noticed outside the eclipses.

\subsection{The close companions}

As seen in Fig. 1, HV 982 has several rather close companions. When possible, Pritchard et al. (1998b) PSF fitted as a group HV 982, the two brightest companions at about 3 arcsec distance (of which the southern actually seems to be two very close stars), and a third at about 6 arcsec distance, except at too large seeing where the contributions of the companions were subtracted assuming known positions and magnitudes.

As our observations were done at much better seeing, we have been able to isolate HV 982 and avoid significant contamination from all the companions in the PSF fits to HV 982. This is demonstrated in Fig. 7. Here the database photometry for the 3 arcsec distance companion (id2673) NNE to HV 982 is plotted versus the orbital phase of HV 982 at the time of the observation, versus the seeing, and versus the sky background level. The companion, which is the brightest one, is about $3.4 \mathrm{mag}(y)$ fainter than HV 982 outside eclipses and its DoPHOT photometry therefore much less accurate. However, we find no correlation between the magnitude of the companion and the orbital phase (i.e. light level) of HV 982 or the sky background level. There is, perhaps, a tendency of slightly fainter companion magnitudes for seeing above about 1.6 arcsec where the scatter on the other hand also increases significantly.

\subsection{Ephemeris and apsidal motion}

One time of each of primary and secondary minimum, derived from the uvby CCD observations, are given in Table 5 together with times published by Gaposhkin (1977) and Pritchard et al. (1998b).

Apsidal motion parameters were derived from a weighted least squares method, following the formalism by Giménez \& Garcia-Palayo (1983) and Giménez \& Bastero (1995). The orbital inclination $i$ and eccentricity $e$ were fixed to the values derived from the photometric analysis; see Sect. 6.4. The results, which are shown in Table 6 and Fig. 8, agree quite well with those obtained by Pritchard et al. (1998b).

For the calculation of phases of the $u v b y$ light curves, we adopt the following linear ephemeris derived for the mid-epoch of the observations from the apsidal motion parameters:

Min $\mathrm{I}=2449340.7172+5.335220 \times E$.

The adopted period is identical to the sidereal period. During the light curve observations from JD 2448970 to JD 2450047 , 

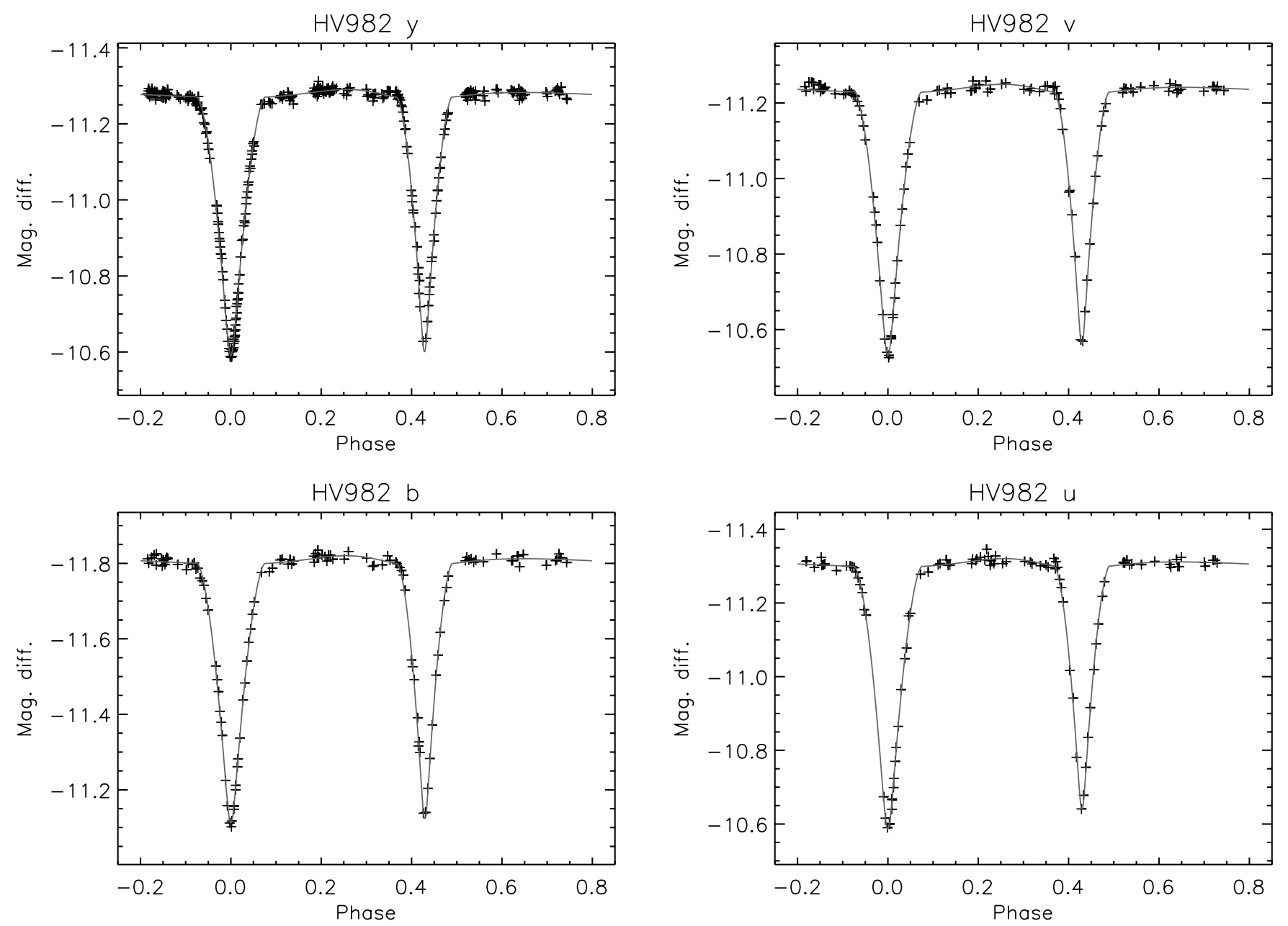

Fig. 6. $u v b y$ light curves of HV 982 (LMC). The theoretical curves correspond to the solutions given in Table 8 .

Table 5. Times of minima for HV 982 (LMC). O-C values (in days) are calculated for the apsidal motion parameters given in Table 6 . References are: G1977, Gaposhkin (1977); P1998, Pritchard et al. (1998b); C2003, this paper. The rms errors of the G1977 times are estimated. Note the perfect agreement between P1998 and C2003 for JD 2449337.

\begin{tabular}{llcrc}
\hline \hline HJD-2 400 000 & rms & Type & O-C & Reference \\
\hline 13946.555 & 0.050 & $\mathrm{P}$ & 0.0290 & $\mathrm{G} 1977$ \\
17590.584 & 0.050 & $\mathrm{P}$ & 0.0350 & - \\
23875.527 & 0.050 & $\mathrm{P}$ & -0.0360 & - \\
25849.645 & 0.050 & $\mathrm{P}$ & 0.0130 & - \\
26060.243 & 0.100 & $\mathrm{~S}$ & 0.0900 & - \\
26412.253 & 0.050 & $\mathrm{~S}$ & -0.0160 & - \\
26577.631 & 0.050 & $\mathrm{~S}$ & -0.0270 & - \\
27786.315 & 0.050 & $\mathrm{P}$ & -0.0380 & - \\
29189.469 & 0.100 & $\mathrm{P}$ & -0.0700 & - \\
29629.338 & 0.050 & $\mathrm{~S}$ & -0.0060 & - \\
31304.630 & 0.050 & $\mathrm{~S}$ & 0.0520 & - \\
32070.603 & 0.050 & $\mathrm{P}$ & 0.0002 & - \\
33153.625 & 0.050 & $\mathrm{P}$ & -0.0420 & - \\
49335.3866 & 0.0004 & $\mathrm{P}$ & 0.0018 & $\mathrm{P} 1998$ \\
49337.6668 & 0.0004 & $\mathrm{~S}$ & -0.0002 & - \\
49337.6670 & 0.0010 & $\mathrm{~S}$ & 0.0018 & $\mathrm{C} 2003$ \\
49340.7172 & 0.0005 & $\mathrm{P}$ & -0.0028 & - \\
50695.8520 & 0.0110 & $\mathrm{P}$ & 0.0086 & $\mathrm{P} 1998$ \\
\hline
\end{tabular}

Table 6. Apsidal motion parameters for HV 982 (LMC).

\begin{tabular}{ll}
\hline \hline Parameter & Value and rms error \\
\hline$i\left(^{\circ}\right)$ & 88.7 (assumed) \\
$e$ & 0.159 (assumed) \\
$T_{0}$ & $2449335.17575 \pm 0.00049$ \\
$P_{\text {anomalistic }}(\mathrm{d})$ & $5.335595 \pm 0.000025$ \\
$P_{\text {sidereal }}(\mathrm{d})$ & 5.335220 \\
$\omega_{0}\left({ }^{\circ}\right)$ & $224.67 \pm 0.15$ \\
$\omega_{1}\left({ }^{\circ} /\right.$ cycle $)$ & $0.00253 \pm 0.00018$ \\
$U(\mathrm{yr})$ & $208 \pm 15$ \\
\hline
\end{tabular}

the longitude of periastron has changed from 224.5 to $225^{\circ} 0$ and the phase of mid secondary eclipse relative to mid primary eclipse from 0.428 to 0.429 . For the light curve analyses, the effect of apsidal motion can be ignored, except for a few points during secondary eclipse from JD 2450041, which have been excluded.

\subsection{Light curve analysis, photometric elements}

Pritchard et al. (1998b) presented photometric elements derived from their CCD light curves, adopting a modified version of the Wilson-Devinney (WD) code (Wilson \& Devinney 1971; Wilson 1994). Light curves in all bands were analyzed 

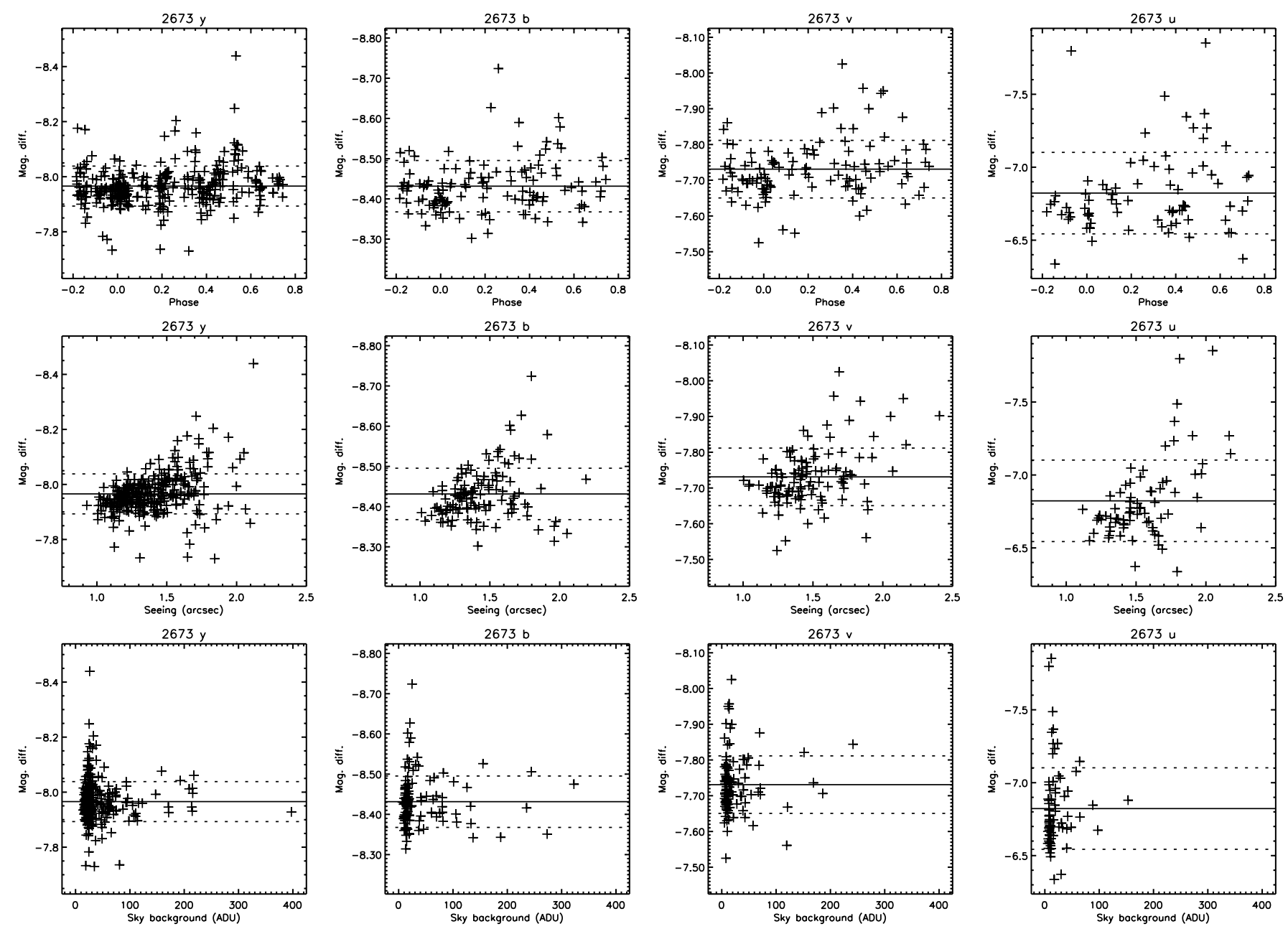

Fig. 7. DoPHOT photometry for the close companion (id2673) about 3 arcsec NNE to HV 982 (LMC); see Fig. 1 . id2673 is about 3.4 mag fainter than HV 982. The full drawn horizontal lines represent the mean magnitudes, and dashed lines are drawn at the 1 sigma levels.

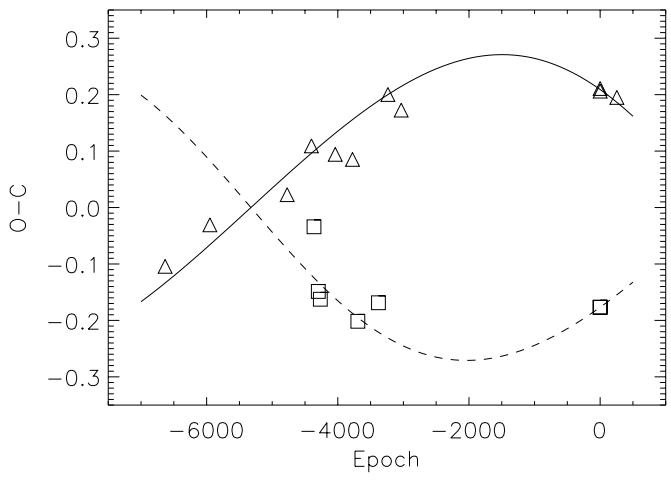

Fig. 8. Apsidal motion for HV 982 (LMC). O-C are the residuals (days) from the linear part of the apsidal motion ephemeris defined by the parameters given in Table 6 . The full and dashed curves represent predictions from the apsidal motion parameters for primary (triangles) and secondary (squares) eclipses, respectively.

simultaneously, and four possible sets of elements were presented, as the differential least squares fit did not converge towards one unique solution. This reflects a situation which is often seen for partially eclipsing systems with fairly identical components, both for circular and eccentric orbits. It is due to significant correlation between several elements. Often the difference between theoretical light curves fitted to the observations for a large range of adopted $k=r_{\mathrm{s}} / r_{\mathrm{p}}$ ( $p$ referring to the component eclipsed at the deeper primary eclipse at phase 0.0 ) are identical within a few mmag. Pritchard et al. (1998b) furthermore did not know the mass ratio $q$ between the components, which is needed for calculation of the stellar shapes. The classical ways to break (part of) the degeneracy are to include independent spectroscopic information on the luminosity ratio (see e.g. Andersen et al. 1983), or - for eccentric systems - independent information on the orbital eccentricity from apsidal motion analyses (see e.g. Clausen et al. 1986).

Fitzpatrick et al. (2002) determined $q=M_{\mathrm{s}} / M_{\mathrm{p}}=1.029 \pm$ 0.027 and the spectroscopic luminosity ratio $L_{\mathrm{s}} / L_{\mathrm{p}}=1.15 \pm$ 0.05 (blue) between the components which, although the accuracy is not as high as could be desired, enabled them to exclude three of the solutions presented by Pritchard et al. (1998b).

In the following, we will present photometric elements derived from WINK (Wood 1971; extensions from Vaz 1984, 1986; Vaz \& Nordlund 1985; Nordlund \& Vaz 1990) and WD (Wilson \& Devinney 1971; Wilson 1994), extensions from Vaz et al. 1995) analyses of the new uvby light curves. Both codes provide physical binary models, adequate for the description 
Table 7. WINK solutions $(y)$ for HV 982 for a range of fixed $k$ values between 0.90 and 1.10. $T_{\mathrm{p}}=24200 \mathrm{~K}$ and $x=0.33$ have been assumed.

\begin{tabular}{lrrrrr}
\hline \hline & $y$ & $y$ & $y$ & $y$ & $y$ \\
\hline$i\left(^{\circ}\right)$ & 88.71 & 88.26 & 88.15 & 88.25 & 88.66 \\
& \pm 14 & \pm 10 & \pm 10 & \pm 11 & \pm 16 \\
$e \cos \omega$ & -0.1115 & -0.1115 & -0.1115 & -0.1116 & -0.1117 \\
& \pm 2 & \pm 2 & \pm 2 & \pm 2 & \pm 2 \\
$e \sin \omega$ & -0.1243 & -0.1194 & -0.1170 & -0.1137 & -0.1124 \\
& \pm 43 & \pm 43 & \pm 43 & \pm 44 & \pm 44 \\
$e$ & 0.1669 & 0.1634 & 0.1617 & 0.1593 & 0.1585 \\
$\omega\left(^{\circ}\right)$ & 228.11 & 226.96 & 226.38 & 225.53 & 225.20 \\
$k$ & 0.90 & 0.95 & 1.00 & 1.05 & 1.10 \\
$r_{\mathrm{p}}$ & 0.2138 & 0.2085 & 0.2033 & 0.1982 & 0.1931 \\
& \pm 7 & \pm 7 & \pm 7 & \pm 7 & \pm 7 \\
$r_{\mathrm{s}}$ & 0.1924 & 0.1981 & 0.2033 & 0.2081 & 0.2125 \\
$r_{\mathrm{p}}+r_{\mathrm{s}}$ & 0.4062 & 0.4066 & 0.4066 & 0.4063 & 0.4056 \\
$T_{\mathrm{s}}(\mathrm{K})$ & 23740 & 23613 & 23572 & 23577 & 23650 \\
& \pm 103 & \pm 104 & \pm 101 & \pm 104 & \pm 104 \\
$J_{\mathrm{s}} / J_{\mathrm{p}}$ & 0.959 & 0.950 & 0.947 & 0.947 & 0.952 \\
$L_{\mathrm{s}} / L_{\mathrm{p}}$ & 0.773 & 0.856 & 0.948 & 1.049 & 1.160 \\
$\sigma(\mathrm{mag})$ & 0.0098 & 0.0099 & 0.0098 & 0.0100 & 0.0100 \\
\hline
\end{tabular}

of HV 982. They differ mainly with respect to the model of the stellar shapes (3-axial ellipsoids with 4th and 5th order terms included versus Roche potentials) and with respect to the elements determined through the differential least squares procedure (e.g. $r_{\mathrm{p}}$ and $k$ versus potentials, and $e \cos \omega$ and $e \sin \omega$ versus $e$ and $\omega$ ). WINK is faster and more used-friendly than WD and therefore well-suited for detailed investigations of multidimensional parameter spaces. An extended IDL-FORTRAN WINK version has been developed by one of us (JVC) for that purpose.

We have selected to perform independent rather than combined analyses of the uvby light curves in order to obtain full understanding of the accuracy to which the elements can be established.

\subsubsection{WINK analyses}

The results from a series of WINK analyses for fixed $k$ values between 0.88 and 1.12 are shown in Fig. 9 and listed in Table 7. An effective temperature of $T_{\mathrm{p}}=24200 \mathrm{~K}$ was assumed in agreement with the combined intrinsic uvby indices given in Table 4 and the calibration by Napiwotzki et al. (1992), as well as with the energy distribution analysis by Fitzpatrick et al. (2002). Surface fluxes for the $u v b y$ bands, as function of $T_{\text {eff }}$ and $\log g$ were based on Kurucz (1992) ATLAS9 atmosphere models (although for Solar composition). Linear limb darkening coefficients $x$ (see Table 8) by Diaz-Cordoves et al. (1995) were adopted, and gravity darkening exponents of 0.25 and bolometric albedos of 1.0 were assumed in accordance with atmospheres in radiative equilibrium. For the model calculation of the stellar deformations, a mass ratio of $q=1.0$ was assumed, and the rotation of the stars was assumed to be pseudosynchronized (rate at periastron). Initially it was settled that no phase shift was needed, i.e. the epoch of the ephemeris given in Eq. (1) is well established.
Table 8. WINK solutions for HV 982. $k=r_{\mathrm{s}} / r_{\mathrm{p}}=1.10$ and $T_{\mathrm{p}}=$ $24200 \mathrm{~K}$ has been assumed.

\begin{tabular}{lrrrr}
\hline \hline & $y$ & $b$ & $v$ & $u$ \\
\hline$i\left(^{\circ}\right)$ & 88.66 & 88.96 & 88.84 & 88.80 \\
& \pm 16 & \pm 32 & \pm 26 & \pm 28 \\
$e \cos \omega$ & -0.1117 & -0.1106 & -0.1106 & -0.1103 \\
& \pm 2 & \pm 4 & \pm 4 & \pm 5 \\
$e \sin \omega$ & -0.1124 & -0.1102 & -0.1065 & -0.1097 \\
& \pm 44 & \pm 75 & \pm 71 & \pm 76 \\
$e$ & 0.1585 & 0.1561 & 0.1535 & 0.1555 \\
$\omega\left(^{\circ}\right)$ & 225.20 & 224.90 & 223.91 & 224.83 \\
$r_{\mathrm{p}}$ & 0.1931 & 0.1929 & 0.1912 & 0.1932 \\
& \pm 7 & \pm 12 & \pm 11 & \pm 12 \\
$r_{\mathrm{s}}$ & 0.2125 & 0.2122 & 0.2103 & 0.2125 \\
$r_{\mathrm{p}}+r_{\mathrm{s}}$ & 0.4056 & 0.4051 & 0.4015 & 0.4057 \\
$x_{\mathrm{p}}=x_{\mathrm{s}}$ & 0.33 & 0.36 & 0.39 & 0.39 \\
$T_{\mathrm{s}}(\mathrm{K})$ & 23650 & 23671 & 23420 & 23468 \\
& \pm 104 & \pm 150 & \pm 138 & \pm 107 \\
$J_{\mathrm{s}} / J_{\mathrm{p}}$ & 0.953 & 0.954 & 0.931 & 0.907 \\
$L_{\mathrm{s}} / L_{\mathrm{p}}$ & 1.160 & 1.160 & 1.134 & 1.105 \\
$\sigma(\mathrm{mag})$ & 0.0100 & 0.0104 & 0.0097 & 0.0100 \\
\hline
\end{tabular}

As seen from the upper panel of Fig. 9, solutions for $k$ between 0.90 and 1.10 fit the observations nearly equally well in all four bands. Several parameters correlate directly with $k$, primarily $e$ (through $e \sin \omega$ ) and $L_{\mathrm{s}} / L_{\mathrm{p}}$, whereas $T_{\mathrm{s}}$ (and thereby the ratio of surface fluxes $J_{\mathrm{s}} / J_{\mathrm{p}}$ ) and the sum of relative radii are nearly constant. Solutions from the four bands agree quite well - the $y$ solution carries highest weight due to the larger number of points and the most complete phase coverage for this band. A luminosity ratio ( $v b y)$ of $L_{\mathrm{s}} / L_{\mathrm{p}}=1.15 \pm 0.05$, as determined by Fitzpatrick et al. (2002), corresponds to $k=1.10 \pm$ 0.03 . We have assumed this value, and solutions for $k=1.10$ are presented in Table 8, and $(\mathrm{O}-\mathrm{C})$ residuals (observed minus calculated mag) are shown in Fig. 10. No systematic effects with phase and seeing are seen, but for an unknown reason a slight tendency of positive residuals (observation fainter than theoretical point) for background levels above about $150 \mathrm{ADU}$ seems to be present. However, the rms errors of the fits are comparable to the photometric errors of the CCD magnitude differences.

\subsubsection{Wilson-Devinney analyses}

For comparison, both with the results from our WINK analyses and with the results by Fitzpatrick et al. (2002), we present in Table 9 a series of WD solutions for the $y$ light curve. The WD solutions were done for very nearly the same ratio of radii $k$ as the WINK solutions given in Table 7, and as for the WINK solutions, a mass ratio of $q=1.0$ and pseudosynchronized rotation of the components have been assumed. $y$ surface fluxes were based on Kurucz (1992) ATLAS9 atmosphere models, a linear limb darkening law with $x=0.33$, gravity exponents of 1.0 (which correspond to input values of 0.25 for WINK), and reflection albedos of 1.0, were adopted.

When comparing the results from the two codes, it should first be noted that in WINK, the relative radii are corrected for the (small) expansion effects from rotation and gravitational 

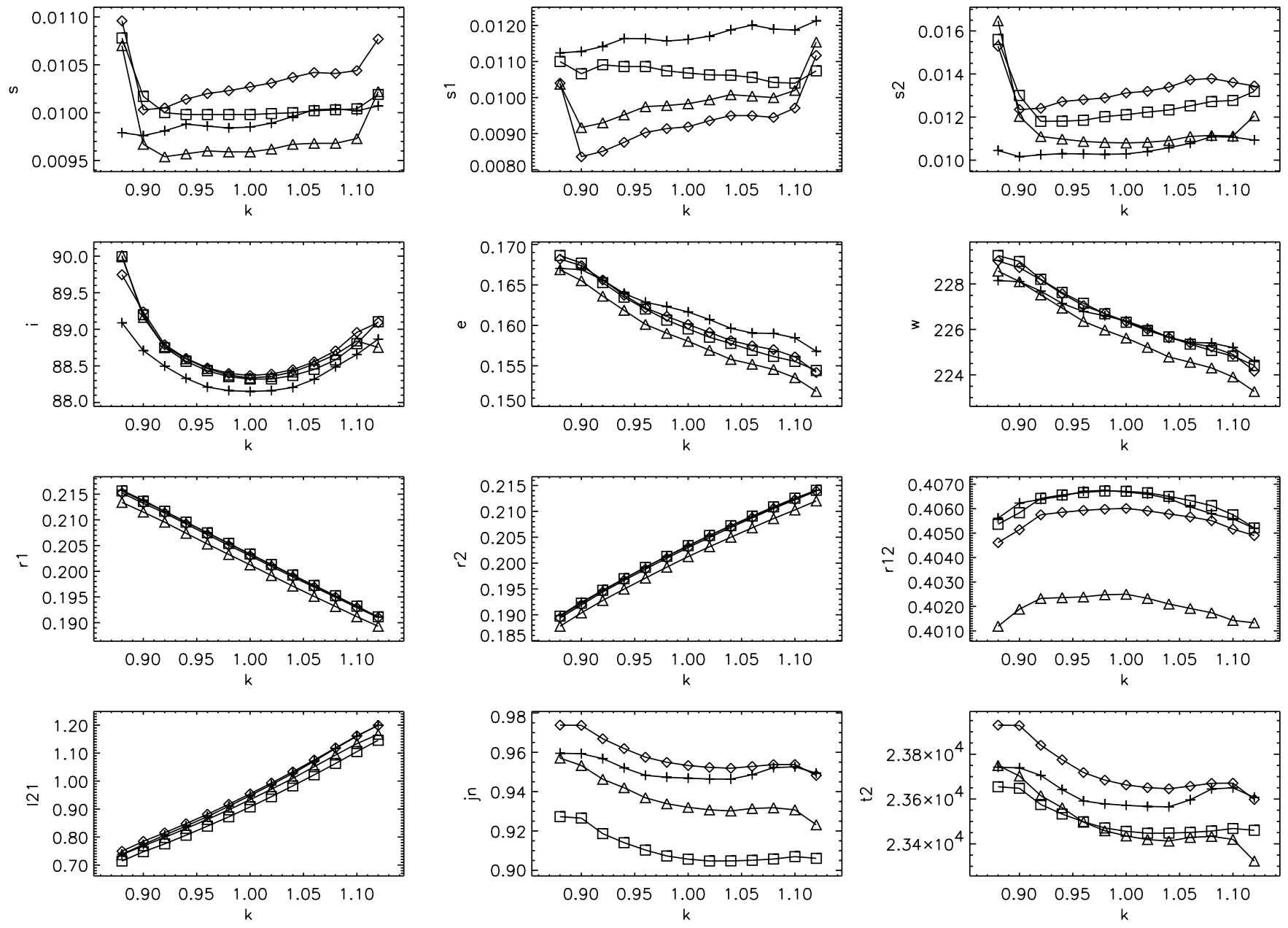

Fig. 9. Results from WINK analyses of HV 982 for adopted $k=r_{\mathrm{s}} / r_{\mathrm{p}}$ values between 0.88 and 1.12. The individual figures show s: rms error (mag), s1: rms error (mag) for primary eclipse, $\mathrm{s} 2$ : rms error (mag) for secondary eclipse, i: orbital inclination $i$, e: orbital eccentricity $e$, w: longitude of periastron $\omega\left(^{\circ}\right), \mathrm{r} 1$ : relative radius for primary component $r_{\mathrm{p}}, \mathrm{r} 2$ : relative radius for secondary component $r_{\mathrm{s}}, \mathrm{r} 12$ : sum of relative radii, 121: luminosity ratio $L_{\mathrm{s}} / L_{\mathrm{p}}$, jn: surface flux ratio, t2: effective temperature of secondary component $T_{\mathrm{s}}(\mathrm{K})$. Symbols are: cross $y$, diamond $b$, triangle $v$, square $u$.

Table 9. WD solutions ( $y$ ) for HV 982 for fixed $\Omega_{\mathrm{p}} . T_{\mathrm{p}}=24200 \mathrm{~K}$ and $x=0.33$ have been assumed. $\Omega$ specify surface potentials and $r$ relative volume radii.

\begin{tabular}{lrrrrr}
\hline \hline & $y$ & $y$ & $y$ & $y$ & $y$ \\
\hline$i\left(^{\circ}\right)$ & 88.71 & 88.34 & 88.26 & 88.32 & 88.74 \\
& \pm 9 & \pm 7 & \pm 5 & \pm 6 & \pm 11 \\
$e$ & 0.1633 & 0.1628 & 0.1606 & 0.1593 & 0.1594 \\
& \pm 20 & \pm 21 & \pm 19 & \pm 19 & \pm 22 \\
$\omega\left(^{\circ}\right)$ & 226.76 & 226.58 & 225.80 & 225.34 & 225.37 \\
& \pm 67 & \pm 72 & \pm 68 & \pm 69 & \pm 79 \\
$\Omega_{\mathrm{p}}$ & 5.900 & 6.020 & 6.125 & 6.240 & 6.385 \\
$\Omega_{\mathrm{s}}$ & 6.488 & 6.362 & 6.253 & 6.108 & 6.015 \\
& \pm 18 & \pm 18 & \pm 16 & \pm 17 & \pm 16 \\
$r_{\mathrm{p}}$ & 0.2172 & 0.2115 & 0.2066 & 0.2016 & 0.1958 \\
$r_{\mathrm{s}}$ & 0.1958 & 0.2007 & 0.2061 & 0.2112 & 0.2155 \\
$k$ & 0.902 & 0.949 & 0.998 & 1.048 & 1.101 \\
$r_{\mathrm{p}}+r_{\mathrm{s}}$ & 0.4130 & 0.4122 & 0.4127 & 0.4128 & 0.4113 \\
$T_{\mathrm{s}}(\mathrm{K})$ & 23536 & 23498 & 23471 & 23452 & 23520 \\
& \pm 75 & \pm 83 & \pm 73 & \pm 65 & \pm 85 \\
$L_{\mathrm{s}} / L_{\mathrm{p}}$ & 0.778 & 0.859 & 0.947 & 1.044 & 1.157 \\
$\sigma(\mathrm{mag})$ & 0.0097 & 0.0097 & 0.0097 & 0.0098 & 0.0099 \\
\hline
\end{tabular}

interaction between the components, whereas such corrections are not applied in the WD code. In the case of HV 982, the corrections are about 0.002 for both components; see Table 10 for further details. Taking this code difference into account, the two binary models are seen to produce practically identical results for HV 982.

\subsubsection{Adopted photometric elements}

From the results of the uvby light curve analyses described above, we derive the mean photometric elements for HV 982 listed in Table 10. Within the errors, our results agree well with those given by Fitzpatrick et al. (2002). Slightly more accurate values have been obtained for the orbital inclination and eccentricity, probably because our uvby photometry is more accurate than that by Pritchard et al. (1998b). Accepting the luminosity ratio derived by Fitzpatrick et al. (2002), both analyses yield relative radii accurate to about $1 \%$, i.e. at the level of the expansion of the components compared to single stars; see Sect. 6.4.2. 

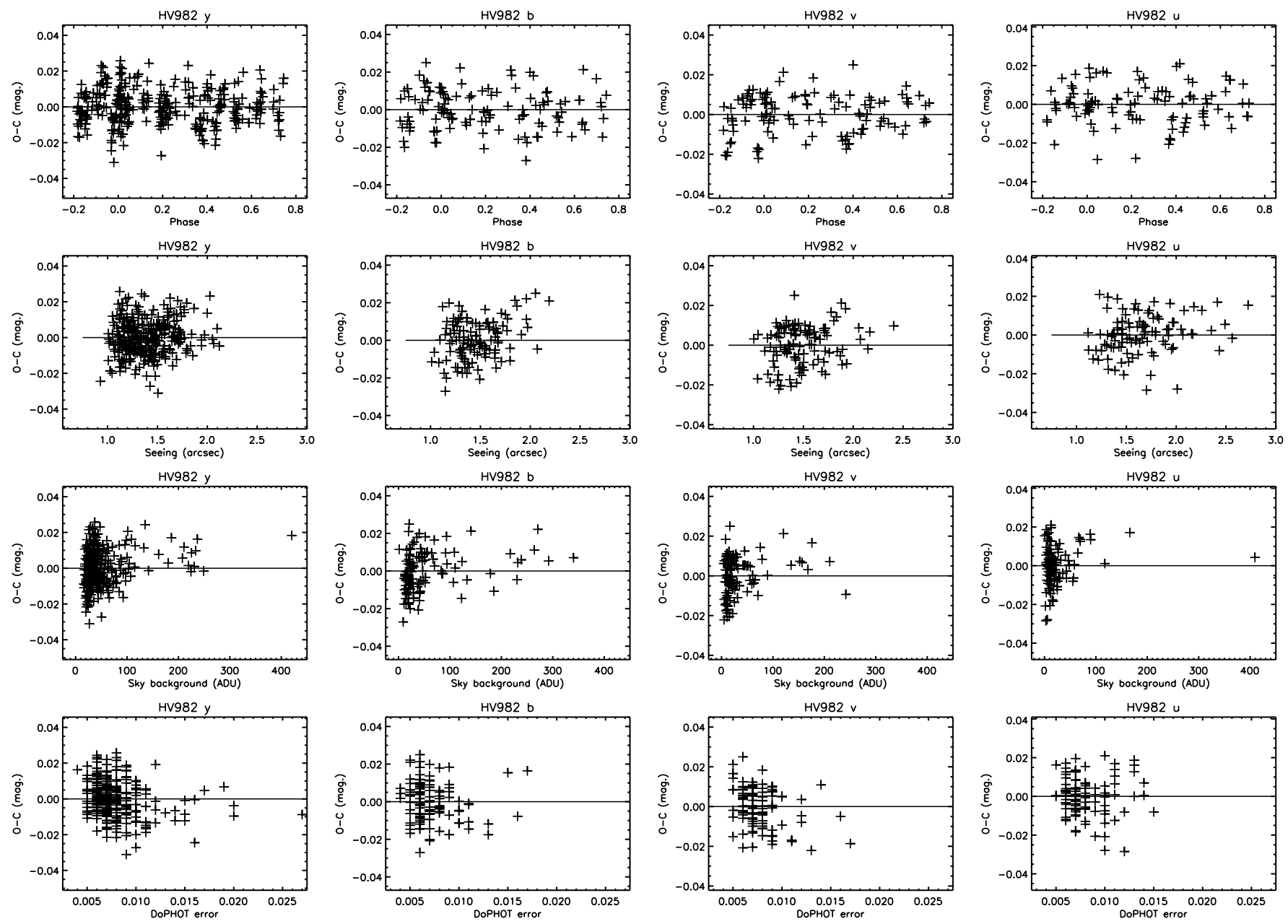

Fig. 10. O-C residuals for the WINK solutions of HV 982 for $k=1.10$ given in Table 8 plotted versus orbital phase, seeing, sky background, and DoPHOT error (mag).

Table 10. Adopted photometric elements for HV 982. $r_{\mathrm{p}}$ and $r_{\mathrm{s}}$ are corrected for expansion due to rotation and tidal effects, the $r_{0}$ results are not. The individual flux and luminosity ratios are based on the mean stellar and orbital parameters.

\begin{tabular}{|c|c|c|c|c|c|c|}
\hline $\bar{i}$ & \multicolumn{6}{|c|}{$88^{\circ} .7 \pm 0.2$} \\
\hline$e \cos \omega$ & \multicolumn{6}{|c|}{$-0.1117 \pm 0.0002($ at JD 2449500$)$} \\
\hline$e \sin \omega$ & \multicolumn{6}{|c|}{$-0.1124 \pm 0.0044$ (at JD 2449500) } \\
\hline$e$ & \multicolumn{6}{|c|}{$0.159 \pm 0.003$} \\
\hline$\omega$ & \multicolumn{6}{|c|}{$225^{\circ} .2 \pm 1.1$ (at JD 2449500) } \\
\hline$r_{\mathrm{p}}$ & \multicolumn{6}{|c|}{$0.194 \pm 0.003$} \\
\hline$r_{\mathrm{s}}$ & \multicolumn{6}{|c|}{$0.214 \pm 0.003$} \\
\hline$r_{\mathrm{p}}+r_{\mathrm{s}}$ & \multicolumn{6}{|c|}{$0.408 \pm 0.003$} \\
\hline$k$ & \multicolumn{6}{|c|}{$1.10 \pm 0.03($ assumed $)$} \\
\hline & $y$ & $b$ & $v$ & $u$ & & \\
\hline$J_{\mathrm{s}} / J_{\mathrm{p}}$ & 0.956 & 0.954 & 0.939 & 0.912 & & \\
\hline$L_{\mathrm{s}} / L_{\mathrm{p}}$ & 1.171 & 1.168 & 1.150 & 1.117 & & \\
\hline \multicolumn{7}{|c|}{ Deformation of the components (WINK model): } \\
\hline & $a$ & $b$ & $c$ & 4th ord. & 5th ord. & $r_{0}$ \\
\hline Star $\mathrm{p}$ & 0.199 & 0.196 & 0.193 & 0.002 & 0.001 & 0.196 \\
\hline Star s & 0.221 & 0.217 & 0.212 & 0.004 & 0.001 & 0.217 \\
\hline
\end{tabular}

\subsection{Absolute dimensions, reddening, and distance}

Preliminary absolute dimensions and distance for HV 982 are given in Table 11 together with photometric data for its components. The results are based on the mean photometric elements listed in Table 10 and the spectroscopic elements by Fitzpatrick et al. (2002). The $u v b y$ indices have been derived from the combined indices at phase 0.25 (Table 3 ) and the luminosity ratios (Table 10). Reddening, interstellar absorption and intrinsic colors are based on the calibration by Crawford (1978). Effective temperatures were calculated from the empirical $[u-b]$ calibration by Napiwotzki et al. (1992), and the well established temperature difference from the light curve solution, and bolometric corrections are based on the calibration by Flower (1996).

The dimensions and temperatures we obtain for HV 982 agree well with those presented by Fitzpatrick et al. (2002), although the photometric data and the methods used for the analyses are different. The distance has been derived from the "classical" formula

$$
\begin{aligned}
V_{0}-M_{V}= & V-A_{V}+5 \times \log \left(R / R_{\odot}\right) \\
& +10 \times \log \left(T_{\text {eff }} / T_{\text {eff } \odot}\right)+B C-M_{\text {bol } \odot}
\end{aligned}
$$

adopting rms errors of $0.01(V), 0.02\left(A_{V}\right)$, and $0.05(B C)$. Our value is slightly larger, but in formal agreement with that 
Table 11. Astrophysical data for HV 982. $M_{\mathrm{bol} \odot}=4.74$ has been assumed (Flower 1996).

\begin{tabular}{lrr}
\hline \hline & Primary & Secondary \\
\hline Absolute dimensions: & & \\
$M / M_{\odot}$ & $11.28 \pm 0.46$ & $11.61 \pm 0.47$ \\
$R / R_{\odot}$ & $7.15 \pm 0.12$ & $7.92 \pm 0.13$ \\
$\log g$ (cgs) & $3.782 \pm 0.023$ & $3.706 \pm 0.022$ \\
& & \\
Photometric data: & & \\
$V$ & 15.490 & 15.318 \\
$(b-y)$ & -0.044 & -0.041 \\
$m_{1}$ & 0.062 & 0.077 \\
$c_{1}$ & 0.012 & 0.027 \\
$E_{(b-y)}$ & 0.068 & 0.082 \\
$A_{V}$ & 0.293 & 0.309 \\
$(b-y)_{0}$ & -0.112 & -0.111 \\
$c_{0}$ & -0.002 & 0.013 \\
{$[u-b]$} & 0.118 & 0.054 \\
$\log T_{\text {eff }}$ & \multicolumn{2}{c}{} \\
$M_{\text {bol }}$ & $4.384 \pm 0.005$ & $4.373 \pm 0.005$ \\
$\log L / L_{\odot}$ & $-5.75 \pm 0.06$ & $-5.86 \pm 0.07$ \\
$B C$ & $4.19 \pm 0.02$ & $4.23 \pm 0.03$ \\
$M_{V}$ & -2.31 & -2.25 \\
$V_{0}-M_{V}$ & $-3.44 \pm 0.06$ & $-3.61 \pm 0.07$ \\
Distance $(\mathrm{kpc})$ & $18.63 \pm 0.08$ \\
\hline
\end{tabular}

obtained by Fitzpatrick et al. (2002) $(50.2 \pm 1.2 \mathrm{kpc})$. However, we expect to refine both absolute dimensions and distance when independent spectroscopic elements, temperatures, luminosity ratios, and chemical abundances become available from the VLT/UVES spectra mentioned in Sect. 1.

Finally, we want briefly to comment on an interesting empirical method presented recently by Salaris \& Groenewegen (2002). The method, which is based on a calibration of Strömgren photometry, can be used for estimation of distances to B-stars in eclipsing binaries, and among various systems, they applied the method to HV 982. For both components, a distance of $48.3 \pm 5.4 \mathrm{kpc}$, close to the result of the binary analysis, was obtained. It is based on the photometry by Pritchard et al. (1998a), and the radii and $V$ data from an earlier version of Fitzpatrick et al. (2002), which was, however, later revised significantly. We have repeated the calculations using the new results listed in Table 11 and derive distances of $56.5 \mathrm{kpc}$ and $56.2 \mathrm{kpc}$, respectively, for the components of HV 982.

\section{HV 12578 (LMC)}

The photographic light curves of HV 12578 (Gaposhkin 1970) indicate a detached system in a circular orbit with components of comparable surface fluxes. The system is relatively bright, $m_{\mathrm{pg}}=15.05$ at maximum light, and both eclipses are about 0.7 mag deep. The orbital period is 2.48 , and from the widths of the eclipses, relative radii of the components of about $0.20-0.25$ are estimated. Although reflection effects seem to be present, we judged from the photographic data that HV 12578 could very well be a promising candidates for determination of accurate dimensions and distances. The field near the binary is not much crowded, but our first CCD exposures showed that HV 12578 has two much fainter neighbours at about 3 arcsec distances; see Fig. 2. At average La Silla seeing they do not cause any problems for the PSF photometry of HV 12578.

\subsection{Light curves}

We observed HV 12578 on 33 nights between JD 2448970 and JD 2450040, and $194(y), 71(b), 65(v)$, and $53(u)$ points were obtained in the four bands. The light curves are given in Tables 29-32 and shown in Fig. 11. Although the phase coverage is not as complete as we would prefer, the $y$ and $b$ light curves are adequate for photometric analyses. The $v$ and $u$ data are more scarce and, besides providing luminosity ratios between the components in these bands, they mainly serve as check data. Typical accuracies per point are given in Table 2.

The new CCD observations reveal that reflection effects are smaller than indicated by the Harvard material, and also that secondary eclipse is shifted slightly from phase 0.50 , i.e. the orbit is eccentric. Both eclipses are deep, about 0.8 mag in all four bands, so the orbital inclination must be very close to $90^{\circ}$ (a similar galactic F-type case is DM Vir; see Andersen et al. 1984). From the CCD observations alone, it is not possible to settle definitively which eclipse is the deeper one. We have therefore decided to keep the $T_{0}$ definition given by the Harvard ephemeris (Payne-Gaposchkin 1971), although the final photometric analysis presented in Sect. 7.3 shows that for this definition, the component eclipsed near phase 0.49 is marginally hotter than the other. We will return to this matter once the mass ratio becomes available from the analysis of the VLT/UVES spectra.

\subsection{Ephemeris}

Three times of primary minimum (at phase 0.00) and one of secondary minimum (near phase 0.49 ), derived from the $u v b y$ CCD observations, are given in Table 12 together with times published by Gaposhkin (1970). Weighted (weight proportional to square of inverse rms) least squares fits to the times of primary and secondary minima yield periods of $2.4803769 \pm$ 0.0000017 and $2.4803730 \pm 0.0000017$, respectively, and nearly identical results are obtained if equal weights are applied. For the calculation of phases of the uvby observations, we adopt

$$
\text { Min I }=\begin{array}{r}
2448971.7778 \\
\pm 6
\end{array} \quad \begin{array}{r}
\text { d } 4803769 \\
\pm 17
\end{array}
$$

The orbital period agrees well with that published by Payne-Gaposchkin (1971), 2.48038, but is much more accurate. The time of secondary eclipse determined from the $u v b y$ observations reveals that the orbit of HV 12578 is slightly eccentric, and this is confirmed by the light curve analysis; see Sect. 7.3. The slight difference in the periods derived from times of primary and secondary eclipses, respectively, could in principle be due to apsidal motion, but since the orbital eccentricity is just about 0.015 this is not very likely, and it can not at all be confirmed from an analysis of the available times of minima. 

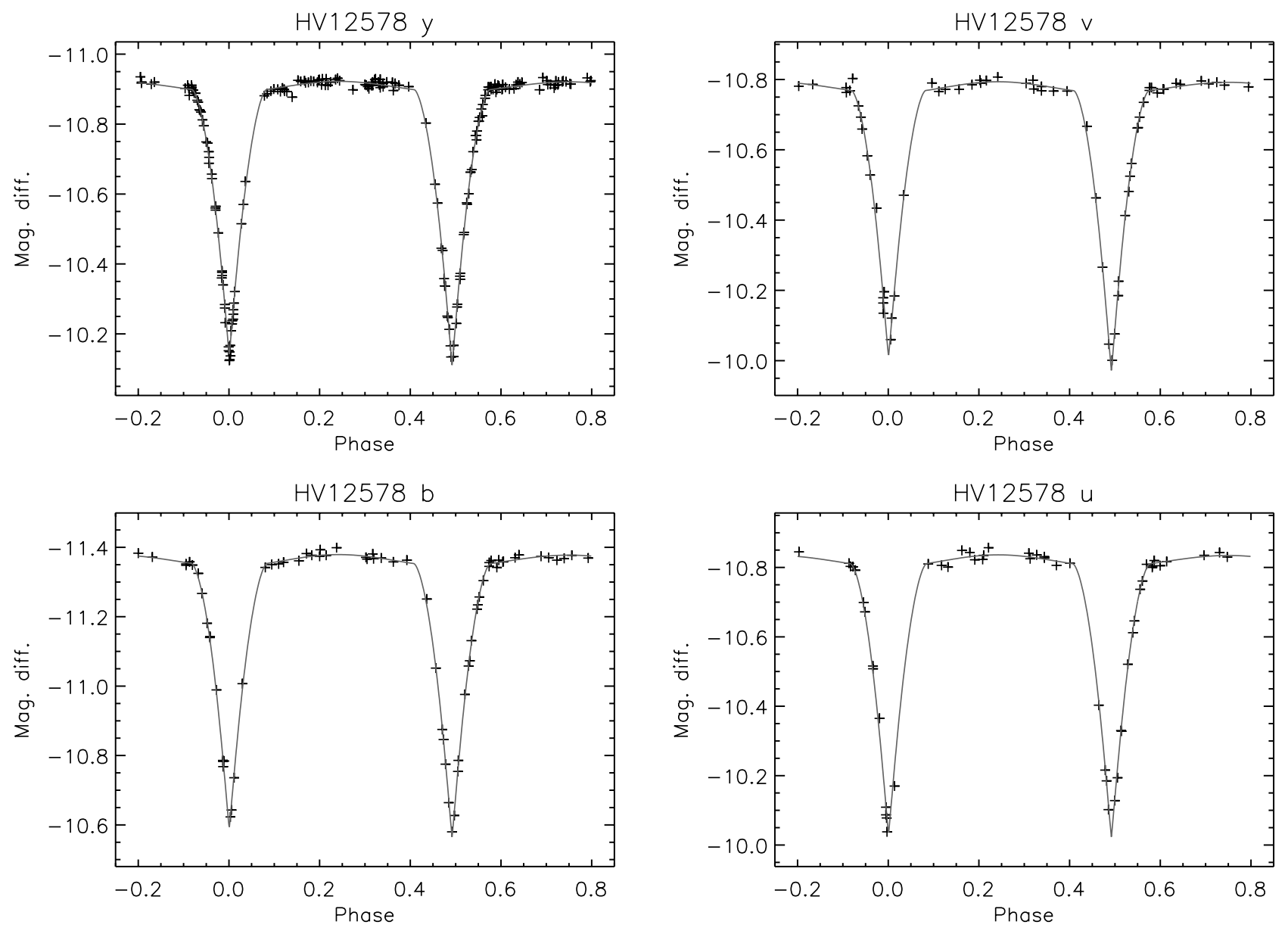

Fig. 11. uvby light curves of HV 12578 (LMC). The theoretical curves correspond to the solutions $(k=1.00)$ given in Table 13 .

Table 12. Times of minima for HV 12578 (LMC). Phases are calculated from the ephemeris given in Eq. (3). References are: G1977, Gaposhkin (1977); C2003, this paper. The rms errors of the G1977 times are estimated. The minimum at JD 2424501.614 listed by G1977 has been omitted.

\begin{tabular}{llcrc}
\hline \hline HJD-2 400 000 & rms & Type & Phase & Reference \\
\hline 16823.638 & 0.050 & $\mathrm{P}$ & 0.010 & $\mathrm{G} 1977$ \\
23681.879 & 0.050 & $\mathrm{P}$ & 0.010 & - \\
23907.499 & 0.050 & $\mathrm{P}$ & -0.028 & - \\
26303.577 & 0.050 & $\mathrm{P}$ & -0.015 & - \\
26328.446 & 0.050 & $\mathrm{P}$ & 0.012 & - \\
27800.507 & 0.050 & $\mathrm{~S}$ & 0.494 & - \\
29881.575 & 0.050 & $\mathrm{~S}$ & 0.507 & - \\
29927.447 & 0.050 & $\mathrm{P}$ & 0.001 & - \\
29994.482 & 0.050 & $\mathrm{P}$ & 0.027 & - \\
30640.598 & 0.050 & $\mathrm{~S}$ & 0.518 & - \\
31496.277 & 0.050 & $\mathrm{~S}$ & 0.498 & - \\
48971.777 & 0.001 & $\mathrm{P}$ & -0.000 & $\mathrm{C} 2003$ \\
49343.835 & 0.001 & $\mathrm{P}$ & 0.000 & - \\
49637.738 & 0.001 & $\mathrm{~S}$ & 0.492 & - \\
50040.825 & 0.010 & $\mathrm{P}$ & 0.002 & - \\
\hline
\end{tabular}

\subsection{Light curve analysis, photometric elements}

For the analyses of the CCD light curves of HV 12578, we have adopted the WINK and Wilson-Devinney models described in Sect. 6.4.
The combined intrinsic uvby indices given in Table 4 and the empirical $[u-b]$ calibration by Napiwotzki et al. (1992) yield a mean $T_{\text {eff }}$ of $22000 \mathrm{~K}$, and since the components have nearly identical surface fluxes, this value has been assigned to the primary component (eclipsed at phase 0.00) and kept fixed throughout the analysis. Linear limb darkening coefficients $x$ (see Table 13) by Diaz-Cordoves et al. (1995) were adopted, and gravity darkening exponents of 0.25 (WINK) and 1.00 (WD), and bolometric albedos of 1.0, were assumed in accordance with atmospheres in radiative equilibrium. For the model calculation of the stellar deformations, a mass ratio of $q=1.0$ was assumed, and the rotation of the stars was assumed to be pseudosynchronized (rate at periastron).

\subsubsection{WINK analyses}

Normally, at least $i, e \cos \omega, e \sin \omega, r_{\mathrm{p}}, T_{\mathrm{s}}$, and possibly $k$, would be kept free and determined through the differential least squares procedure, but initial tests showed that this approach is not possible for HV 12578, primarily because $i$ is very close to $90^{\circ}$. We have therefore calculated grids of solutions for adopted values of $i$, each of them for a range of adopted $k$ values centered at 1.0. Figure 12 illustrates the results from such WINK solutions for the $y$ light curve. It is seen that the best fits are obtained for $i$ between $89^{\circ} .8$ and 90.0 and $k$ between 0.98 and 1.02 . For a given $k$, most other parameters - especially the individual radii - are very little dependent on $i$ within the range 
Table 13. WINK solutions for HV 12578 for $k=r_{\mathrm{s}} / r_{\mathrm{p}}$ values of $0.98,1.00$, and 1.02. $i=90.0$ and $T_{\mathrm{p}}=22000 \mathrm{~K}$ were adopted. For the $b, v$, and $u$ solutions, $e \cos \omega$ and $e \sin \omega$ were fixed at the $y$ results.

\begin{tabular}{|c|c|c|c|c|c|c|c|c|c|c|c|c|}
\hline & $y$ & $\bar{b}$ & $\bar{v}$ & $\bar{u}$ & $\bar{y}$ & $\bar{b}$ & $\overline{\bar{v}}$ & $\bar{u}$ & $y$ & $\overline{\bar{b}}$ & $\overline{\bar{v}}$ & $u$ \\
\hline $\bar{k}$ & 0.98 & 0.98 & 0.98 & 0.98 & 1.00 & 1.00 & 1.00 & 1.00 & 1.02 & 1.02 & 1.02 & 1.02 \\
\hline$e \sin \omega$ & 0.00648 & 0.00648 & 0.00648 & 0.00648 & 0.00745 & 0.00745 & 0.00745 & 0.00745 & 0.01076 & 0.01076 & 0.01076 & 0.01076 \\
\hline$e \cos \omega$ & $\begin{array}{r} \pm J J 1 \\
-0.01341 \\
\pm 31\end{array}$ & -0.01341 & -0.01341 & -0.01341 & $\begin{array}{r} \pm J 40 \\
-0.01341 \\
\pm 30\end{array}$ & -0.01341 & -0.01341 & -0.01341 & $\begin{array}{r} \pm J J 5 \\
-0.01341 \\
\pm 31\end{array}$ & -0.01341 & -0.01341 & -0.01341 \\
\hline$e$ & 0.0149 & 0.0149 & 0.0149 & 0.0149 & 0.0153 & 0.0153 & 0.0153 & 0.0153 & 0.0172 & 0.0172 & 0.0172 & 0.0172 \\
\hline$\omega\left(\left(^{\circ}\right)\right.$ & 154.2 & 154.2 & 154.2 & 154.2 & 150.9 & 150.9 & 150.9 & 150.9 & 141.3 & 141.3 & 141.3 & 141.3 \\
\hline$r_{\mathrm{p}}$ & $\begin{array}{r}0.2450 \\
\pm 10\end{array}$ & $\begin{array}{r}0.2479 \\
\pm 13\end{array}$ & $\begin{array}{r}0.2442 \\
\pm 2 £\end{array}$ & $\begin{array}{r}0.2497 \\
\pm 25\end{array}$ & $\begin{array}{r}0.2426 \\
\pm 10\end{array}$ & $\begin{array}{r}0.2455 \\
\pm 14\end{array}$ & $\begin{array}{r}0.2419 \\
\pm 20\end{array}$ & $\begin{array}{r}0.2472 \\
\pm 23\end{array}$ & $\begin{array}{r}0.2402 \\
\pm 10\end{array}$ & $\begin{array}{r}0.2430 \\
\pm 13\end{array}$ & $\begin{array}{r}0.2395 \\
\pm 22\end{array}$ & $\begin{array}{r}0.2447 \\
\pm 23\end{array}$ \\
\hline$r_{\mathrm{s}}$ & 0.2401 & 0.2429 & 0.2394 & 0.2447 & 0.2426 & 0.2455 & 0.2419 & 0.2472 & 0.2450 & 0.2478 & 0.2443 & 0.2496 \\
\hline$r_{\mathrm{p}}+r_{\mathrm{s}}$ & 0.4851 & 0.4908 & 0.4836 & 0.4944 & 0.4852 & 0.4910 & 0.4838 & 0.4944 & 0.4852 & 0.4908 & 0.4838 & 0.4943 \\
\hline$x_{\mathrm{p}}=x_{\mathrm{s}}$ & 0.32 & 0.37 & 0.39 & 0.39 & 0.32 & 0.37 & 0.39 & 0.39 & 0.32 & 0.37 & 0.39 & 0.39 \\
\hline$T_{\mathrm{s}}(\mathrm{K})$ & $\begin{array}{r}22328 \\
+76\end{array}$ & $\begin{array}{r}22328 \\
+100\end{array}$ & $\begin{array}{r}22447 \\
+151\end{array}$ & $\begin{array}{r}22079 \\
+107\end{array}$ & $\begin{array}{r}22332 \\
+75\end{array}$ & $\begin{array}{r}22295 \\
+100\end{array}$ & $\begin{array}{r}22430 \\
+151\end{array}$ & $\begin{array}{r}22073 \\
+108\end{array}$ & $\begin{array}{r}22277 \\
+76\end{array}$ & $\begin{array}{r}22289 \\
+100\end{array}$ & $\begin{array}{r}22409 \\
+151\end{array}$ & $\begin{array}{r}22058 \\
+108\end{array}$ \\
\hline$J_{\mathrm{s}} / J_{\mathrm{p}}$ & 1.027 & 1.028 & 1.041 & 1.010 & 1.027 & 1.025 & 1.039 & 1.009 & 1.023 & 1.024 & 1.037 & 1.007 \\
\hline$L_{\mathrm{s}} / L_{\mathrm{p}}$ & 0.986 & 0.987 & 0.999 & 0.971 & 1.028 & 1.026 & 1.040 & 1.011 & 1.066 & 1.068 & 1.081 & 1.051 \\
\hline$\sigma(\mathrm{mag})$ & 0.0109 & 0.0093 & 0.0148 & 0.0157 & 0.0107 & 0.0094 & 0.0149 & 0.0159 & 0.0111 & 0.0093 & 0.0149 & 0.0160 \\
\hline
\end{tabular}

Table 14. WD solutions $(y)$ for HV 12578 for fixed $\Omega_{\mathrm{p}} . i=90.0$, $T_{\mathrm{p}}=22000 \mathrm{~K}$ and $x=0.32$ have been assumed.

\begin{tabular}{lrrr}
\hline \hline & $y$ & $y$ & $y$ \\
\hline$e$ & 0.0157 & 0.0153 & 0.0171 \\
& \pm 12 & \pm 14 & \pm 20 \\
$\omega\left(^{\circ}\right)$ & 150.7 & 150.3 & 139.4 \\
& \pm 6.9 & \pm 8.0 & \pm 6.9 \\
$\Omega_{\mathrm{p}}$ & 5.040 & 5.120 & 5.200 \\
$\Omega_{\mathrm{s}}$ & 5.117 & 5.126 & 5.138 \\
& \pm 18 & \pm 16 & \pm 17 \\
$r_{\mathrm{p}}$ & 0.2513 & 0.2462 & 0.2414 \\
$r_{\mathrm{s}}$ & 0.2464 & 0.2458 & 0.2452 \\
$k$ & 0.981 & 0.998 & 1.016 \\
$r_{\mathrm{p}}+r_{\mathrm{s}}$ & 0.4977 & 0.4920 & 0.4866 \\
$T_{\mathrm{s}}(\mathrm{K})$ & 22502 & 22512 & 22461 \\
& \pm 54 & \pm 57 & \pm 57 \\
$L_{\mathrm{s}} / L_{\mathrm{p}}$ & 0.983 & 1.020 & 1.052 \\
$\sigma(\mathrm{mag})$ & 0.0115 & 0.0114 & 0.0121 \\
\hline
\end{tabular}

mentioned above. Similar analyses for the other three bands support these findings, but are less accurate due to the fewer observations.

Since $i=90^{\circ} 0$ formally gives the best fit we adopt this value, and in Fig. 13 solutions from all four light curves are shown. The $y$ and $b$ results agree well, but due to the incomplete phase coverage in $v$ and $u$, the elements from these two bands are less reliable. For the final solutions, we have chosen for all bands to fix $e$ and $\omega$ at the $y$ results. Such solution for $k=0.98$, 1.00, and 1.02 are presented in Table 13, and the $\mathrm{O}-\mathrm{C}$ residuals for the $k=1.00$ solutions are shown in Fig. 14. The uvby solutions agree quite well, and no systematic trends exist in the residuals. In all four bands, the component eclipsed near phase 0.49 and by us referred to as the secondary, is slightly more luminous than the (primary) component eclipsed at phase 0.00 .

\subsubsection{Wilson-Devinney analyses}

For comparison, a few WD solutions for the $y$ light curve, done for very nearly the same ratio of radii $k$ as the WINK solutions listed in Table 13, are given in Table 14. Taking into account the effect from the different treatment of the expansion effects on the resulting radii, explained in Sect. 6.4.2, we find only small differences between the WINK and WD results. We notice a slightly higher surface flux ratio (via $T_{\mathrm{s}}$ ) for the WD solution.

\subsubsection{Adopted photometric elements}

The adopted mean photometric elements are listed in Table 15. The components are very nearly identical, and as seen, their relative sizes are slightly larger than for HV 982. Relative radii accurate to $1 \%$ have been established, together with well established elements for the very slightly eccentric orbit. Thus, when the results from the analysis of the VLT/UVES spectra are available, we expect to be able to present accurate astrophysical parameters and distance for HV 12578.

\section{HV 1433 (SMC)}

At the time of the uvby observations, only about 30 SMC eclipsing binaries were known, and the selection of candidates therefore more difficult than for LMC. Also, the quality of the photographic light curves from the Harvard survey (Gaposhkin 1977) is rather low, and it was not possible to identify any clearly well-detached systems.

CCD photometry has been obtained for several Harvard SMC systems (HV 1620, Pritchard et al. 1998a; HV 1761, Duncan et al. 1993; HV 1876, Jensen et al. 1988; HV 2016, Jensen et al. 1988; HV 2208, West et al. 1992; HV 2226, Jensen et al. 1988; Bell et al. 1991), and we initially included four additional in our project, HV 1346, HV 1433, HV 1597, 

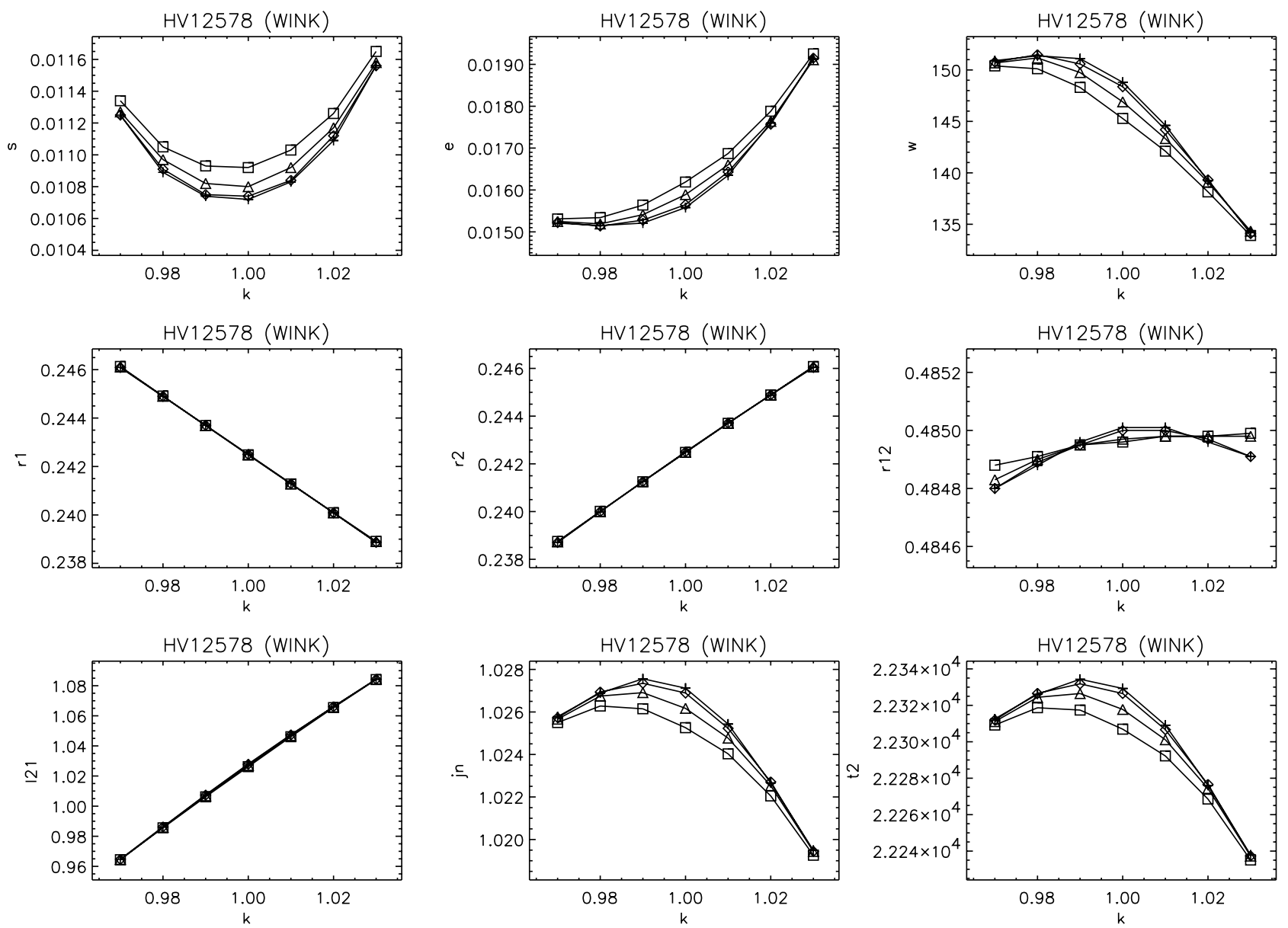

Fig. 12. Results from WINK analyses of the $y$ light curve of HV 12578 for adopted $i$ values between 89.7 and 90.0 , and $k=r_{\mathrm{s}} / r_{\mathrm{p}}$ values between 0.97 and 1.03. A constant phase shift of 0.00065 has been used. The individual figures show s: rms error (mag), e: orbital eccentricity $e$, w: longitude of periastron $\omega\left(^{\circ}\right), \mathrm{r} 1$ : relative radius for primary component $r_{\mathrm{p}}, \mathrm{r} 2$ : relative radius for secondary component $r_{\mathrm{s}}, \mathrm{r} 12$ : sum of relative radii, 121: luminosity ratio $L_{\mathrm{s}} / L_{\mathrm{p}}$, jn: surface flux ratio, t2: effective temperature of secondary component $T_{\mathrm{s}}(\mathrm{K})$. Symbols are: cross $i=90^{\circ}$, diamond $i=89^{\circ} .9$, triangle $i=89^{\circ} .8$, square $i=89^{\circ} .7$.

and HV 11284. For HV $1346(P=66.9)$ and HV 1597 $(P=4.2)$, rather few observations were obtained, partial light curves were obtained for HV 1433, and for HV 11284 we obtained complete light curves. In the present paper, we will only include the HV 1433 and HV 11284 observations.

As mentioned in Sect. 2, HV 1433 is located in the southern part of the central region of SMC, just south of the OGLE - II SMC_SC4 field. Except for a 1.8 mag (y) fainter star about 3". 7 east of HV 1433 (see Fig. 3), which causes no problem for the PSF photometry, the surrounding field is quite clean.

Apart from the photographic Harvard light curve, no earlier observations of HV 1433 have been published.

\subsection{Light curves}

HV 1433 was observed on 19 nights between JD 2448971 and JD 2450040, and 84(y), $38(b), 33(v)$, and $30(u)$ points were obtained in the four bands. The light curves are given in Tables 33-36 and shown in Fig. 15. Typical accuracies per point are given in Table 2.

Unfortunately, the coverage of the light curves is far from complete, and it is problematic to evaluate from the light curve
Table 15. Adopted photometric elements for HV 12578. $r_{\mathrm{p}}$ and $r_{\mathrm{s}}$ are corrected for expansion due to rotation and tidal effects, the $r_{0}$ result (both components) is not. The individual flux and luminosity ratios are based on the mean stellar and orbital parameters.

\begin{tabular}{|c|c|c|c|c|c|c|}
\hline $\bar{i}$ & \multicolumn{6}{|c|}{$90^{\circ} 0 \pm 0.2$} \\
\hline$e \cos \omega$ & \multicolumn{6}{|c|}{$-0.01341 \pm 0.00030$} \\
\hline$e \sin \omega$ & \multicolumn{6}{|c|}{$0.00745 \pm 0.00200$} \\
\hline$e$ & \multicolumn{6}{|c|}{$0.0153 \pm 0.0010$} \\
\hline$\omega$ & \multicolumn{6}{|c|}{$151.0 \pm 6.5$} \\
\hline$r_{\mathrm{p}}$ & \multicolumn{6}{|c|}{$0.2425 \pm 0.0025$} \\
\hline$r_{\mathrm{s}}$ & \multicolumn{6}{|c|}{$0.2425 \pm 0.0025$} \\
\hline $\begin{array}{l}r_{\mathrm{p}}+r_{\mathrm{s}} \\
k\end{array}$ & \multicolumn{6}{|c|}{$0.4850 \pm 0.0035$} \\
\hline & $y$ & $b$ & $v$ & $u$ & & \\
\hline$J_{\mathrm{s}} / J_{\mathrm{p}}$ & 1.027 & 1.026 & 1.039 & 1.011 & & \\
\hline$L_{\mathrm{s}} / L_{\mathrm{p}}$ & 1.028 & 1.027 & 1.040 & 1.013 & & \\
\hline \multicolumn{7}{|c|}{ Deformation of the components (both; WINK model): } \\
\hline & $a$ & $b$ & $c$ & 4th ord. & 5th ord. & $r_{0}$ \\
\hline & 0.2498 & 0.2442 & 0.2408 & 0.0037 & 0.0009 & 0.2449 \\
\hline
\end{tabular}



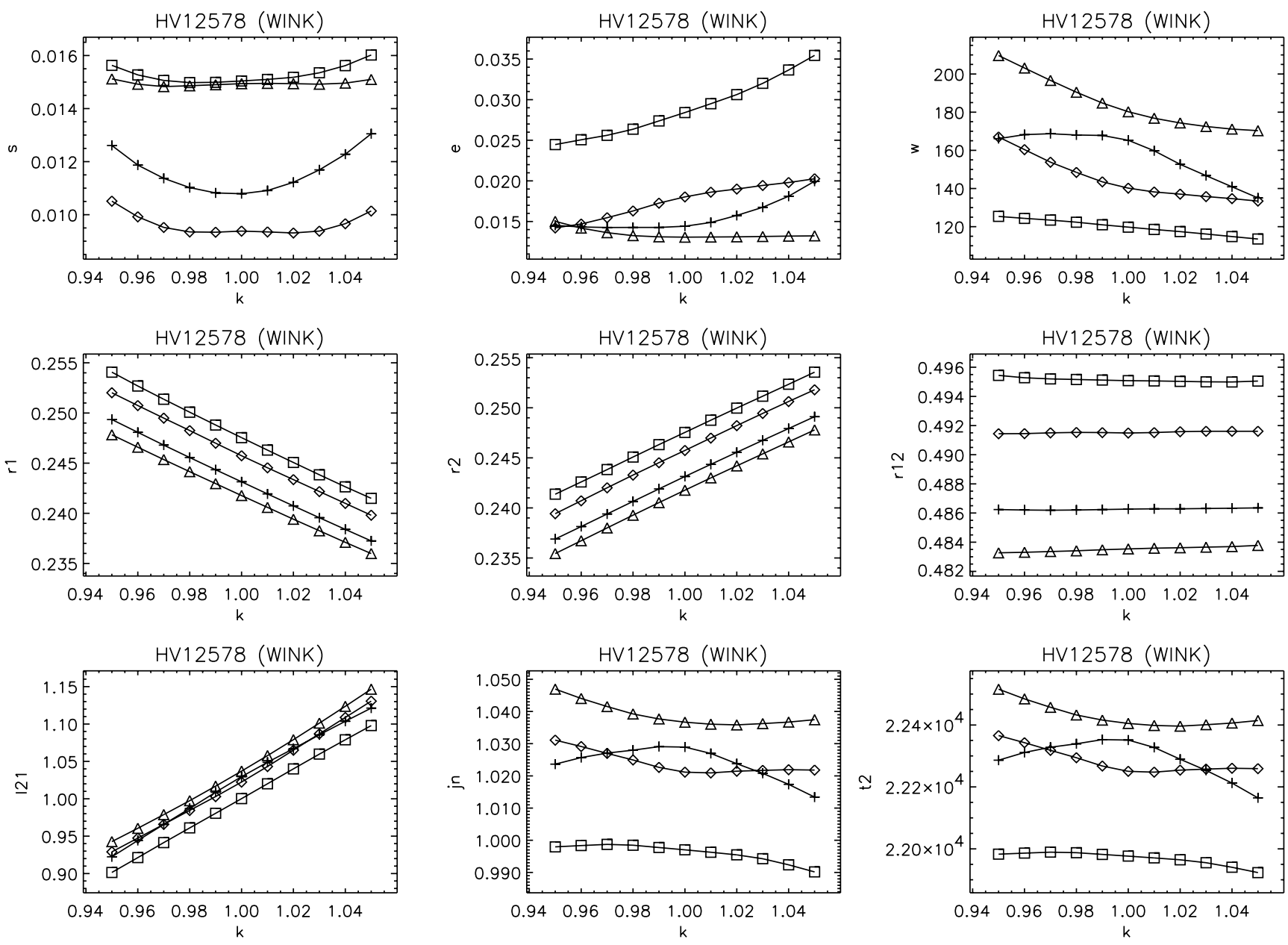

Fig. 13. Results from WINK analyses of HV 12578 for an adopted orbital inclination $i=90.0$, and $k=r_{\mathrm{s}} / r_{\mathrm{p}}$ values between 0.95 and 1.05 . The individual figures show s: rms error (mag), e: orbital eccentricity $e$, w: longitude of periastron $\omega\left({ }^{\circ}\right), \mathrm{r} 1$ : relative radius for primary component $r_{\mathrm{p}}, \mathrm{r} 2$ : relative radius for secondary component $r_{\mathrm{s}}, \mathrm{r} 12$ : sum of relative radii, 121 : luminosity ratio $L_{\mathrm{s}} / L_{\mathrm{p}}$, jn: surface flux ratio, t2: effective temperature of secondary component $T_{\mathrm{s}}(\mathrm{K})$. Symbols are: cross $y$, diamond $b$, triangle $v$, square $u$.

Table 16. Times of minima for HV 1433 (LMC). Phases are calculated from the ephemeris given in Eq. (4). References are: SAO9, PayneGaposchkin \& Gaposchkin (1966); C2003, this paper.

\begin{tabular}{llccc}
\hline \hline HJD-2 400 000 & rms & Type & Phase & Reference \\
\hline 24431.682 & & $\mathrm{P}$ & 0.0000 & SAO9 \\
50037.6300 & 0.0050 & $\mathrm{P}$ & 0.0000 & $\mathrm{C} 2003$ \\
50038.6544 & 0.0005 & $\mathrm{~S}$ & 0.5004 & - \\
50040.7055 & 0.0010 & $\mathrm{~S}$ & 0.5024 & - \\
\hline
\end{tabular}

shapes if HV 1433 could maybe be detached, or if it is a semi-detached system, possibly with the secondary component filling its Roche lobe, like e.g. HV 2208 and HV 2226. However, the latter appears to be the most likely case. As the depth of secondary eclipse is only about half of that of primary, the temperatures of the components must be quite different. The orbit appears to be circular. In Sect. 8.3 we will sketch possible photometric solutions.

\subsection{Ephemeris}

One time of primary minimum and two of secondary minimum, derived from the uvby CCD observations, are given in Table 16 together with the epoch of the ephemeris by Payne-Gaposchkin \& Gaposchkin (1966). We adopt the following ephemeris, derived from the new time of primary minimum and the old epoch

$$
\text { Min I }=\begin{array}{r}
2450037.630 \\
\pm 5
\end{array} \quad \begin{array}{r}
2.047002 \\
\pm 5
\end{array}
$$

The period is slightly larger than that derived from the photographic Harvard observations (2.046987).

\subsection{Light curve analysis, photometric elements}

We have applied only the Wilson-Devinney model for the photometric analysis of HV 1433, since its components are expected to be larger and more deformed than appropriate for the WINK model; see Sect. 6.4 for details on the actual WD code. A semi-detached configuration with the secondary filling its Roche lobe has been assumed, since initial tests showed that detached models converges towards this configuration.

The standard $u v b y$ indices for the combined light of the components, listed in Table 4, correspond to an effective temperature of about $23000 \mathrm{~K}$ (Napiwotzki et al. 1992), which has then been adopted for the primary component, although it 

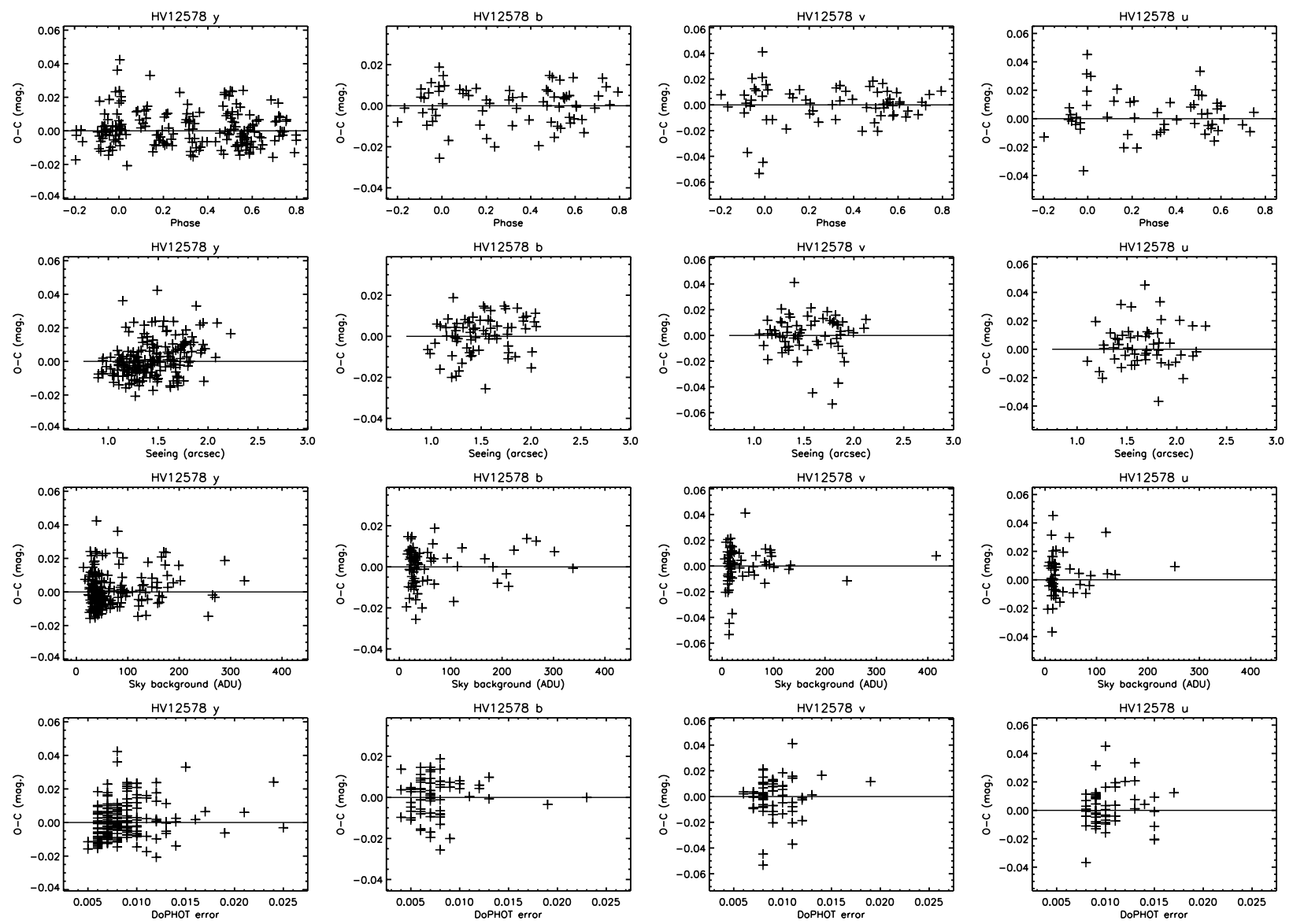

Fig. 14. O-C residuals for the WINK solutions of HV 12578 for $i=90^{\circ}$ and $k=1.00$ given in Table 13 plotted versus orbital phase, seeing, sky background, and DoPHOT error (mag).

might be slightly on the low side due to the effect on the indices of the cooler component. Linear limb darkening coefficients by Diaz-Cordoves et al. (1995) were adopted together with gravity exponents and reflection albedos of 1.0. A circular orbit and synchronized rotation of the components were assumed.

Solutions for a range of fixed mass ratios indicate that values below 0.4 and above 0.6 are not very likely. In Table 17 we present a few solutions within the remaining range for which acceptable fits are reached. Definitive solutions can not be obtained from the incomplete light curves, but relative radii of around 0.34 (primary) and 0.32 (secondary), a temperature difference between the components of about $7000 \mathrm{~K}$, and an orbital inclination of about $86^{\circ}$ are estimated.

\subsection{Discussion}

Due to its brightness, its position within the SMC, and its uncomplicated surrounding field, HV 1433 is an interesting target for future photometric and spectroscopic observations and studies, primarily for distance determinations. On the other hand, many of the new eclipsing binaries in the central region of SMC, now known from the OGLE - II project, could just as well be considered. They include about 150 detached systems, most of which are, however, much fainter than HV 1433 and therefore in general more difficult to observe accurately enough.

\section{HV 11284 (SMC)}

As mentioned in Sect. 2, HV 11284 is located slightly west of the optical center of SMC in the OGLE - II field SMC_SC5 and is identical to the OGLE - II eclipsing binary SMC_SC5_140701. We selected HV 11284 as one of the most promising systems among the few Harvard candidates available and obtained complete CCD light curves 1992-94. In addition, BVI light curves from 1997-98 are now also available from OGLE - II (Udalski et al. 1998b).

Being nearer to the SMC center, the HV 11284 field is more crowded than that of HV 1433 with a higher density of faint stars. There are no bright, close companions to HV 11284, but the shape of its image on CCD exposures obtained at about 1 arcsec seeing indicates the presence of a possible very close, but also quite faint companion slightly to the east; see Fig. 4. If real, it is included in the DoPHOT photometry of HV 11284 and therefore may add a small amount of 3rd light to the light curve data. We estimate that it is at least 3-4 mag fainter than HV 11284 at maximum light, leading to an upper level of about $6 \%$ of possible 3 rd light $\left(L_{3} /\left(L_{\mathrm{p}}+L_{\mathrm{S}}\right)\right)$.

\subsection{Light curves}

HV 11284 was observed on 34 nights between JD 2448971 and JD 2449638, and $219(y), 81(b), 70(v)$, and $63(u)$ points 

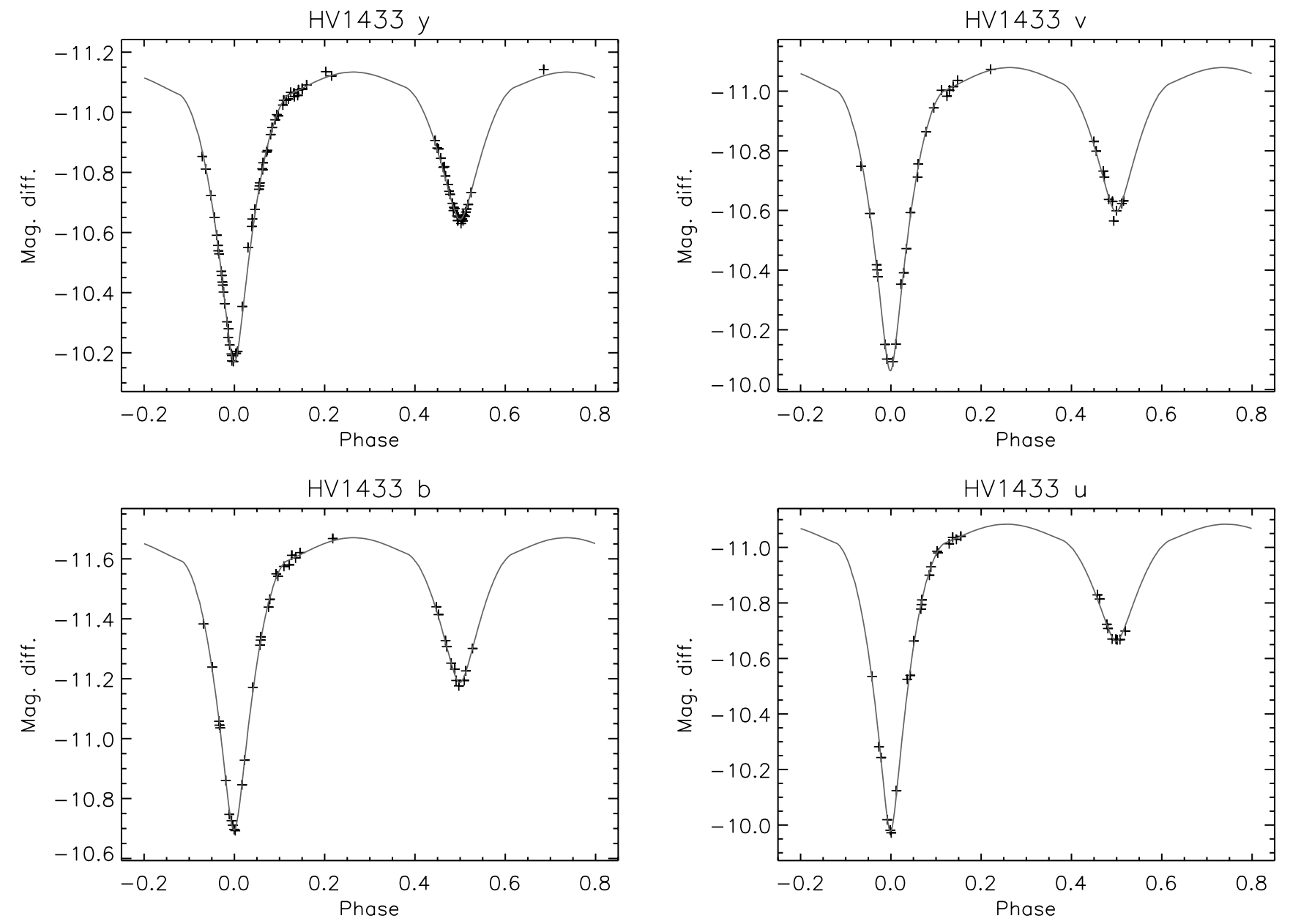

Fig. 15. uvby light curves of HV 1433 (SMC). The theoretical curves correspond to the solutions $(q=0.50)$ given in Table 17 .

Table 17. WD solutions for HV 1433 for adopted mass ratios between 0.4 and 0.6 . A semi-detached configuration is assumed, and $T_{\mathrm{p}}=$ $23000 \mathrm{~K}$ has been adopted throughout.

\begin{tabular}{lrrrrrr}
\hline \hline & $y$ & $y$ & $b$ & $v$ & $u$ & $y$ \\
\hline$q=m_{\mathrm{s}} / m_{\mathrm{p}}$ & 0.40 & 0.50 & 0.50 & 0.50 & 0.50 & 0.60 \\
$i\left(^{\circ}\right)$ & 88.37 & 86.17 & 84.43 & 86.15 & 86.68 & 84.80 \\
& \pm 20 & \pm 15 & \pm 18 & \pm 37 & \pm 44 & \pm 10 \\
$\Omega_{\mathrm{p}}$ & 3.3394 & 3.4093 & 3.5562 & 3.4710 & 3.4474 & 3.6630 \\
& \pm 84 & \pm 192 & \pm 398 & \pm 557 & \pm 425 & \pm 201 \\
$\Omega_{\mathrm{s}}$ & 2.6781 & 2.8758 & 2.8758 & 2.8758 & 2.8758 & 3.0624 \\
$r_{\mathrm{p}}$ & 0.347 & 0.352 & 0.334 & 0.344 & 0.347 & 0.333 \\
$r_{\mathrm{s}}$ & 0.303 & 0.321 & 0.321 & 0.321 & 0.321 & 0.336 \\
$x_{\mathrm{p}}$ & 0.34 & 0.34 & 0.38 & 0.40 & 0.40 & 0.34 \\
$x_{\mathrm{s}}$ & 0.37 & 0.37 & 0.43 & 0.46 & 0.43 & 0.37 \\
$T_{\mathrm{s}}(\mathrm{K})$ & 16157 & 16150 & 16425 & 16577 & 16595 & 16180 \\
& \pm 67 & \pm 54 & \pm 70 & \pm 102 & \pm 117 & \pm 51 \\
$L_{\mathrm{s}} / L_{\mathrm{p}}$ & 0.455 & 0.494 & 0.539 & 0.494 & 0.356 & 0.600 \\
$\sigma(\mathrm{mag})$ & 0.0134 & 0.0111 & 0.0098 & 0.0148 & 0.0150 & 0.0120 \\
\hline
\end{tabular}

were obtained in the four bands. The light curves are given in Tables 37-40 and shown in Fig. 16. Typical accuracies per point are given in Table 2.

As seen from the relatively broad eclipses and the light variations outside eclipses, HV 11284 consists of two rather close and significantly deformed components. It is, however, not quite obvious if the system is detached or semi-detached. The eclipses differ significantly in depths, and the secondary component eclipsed at phase 0.5 is clearly much cooler than the primary. The orbit appears to be circular. 

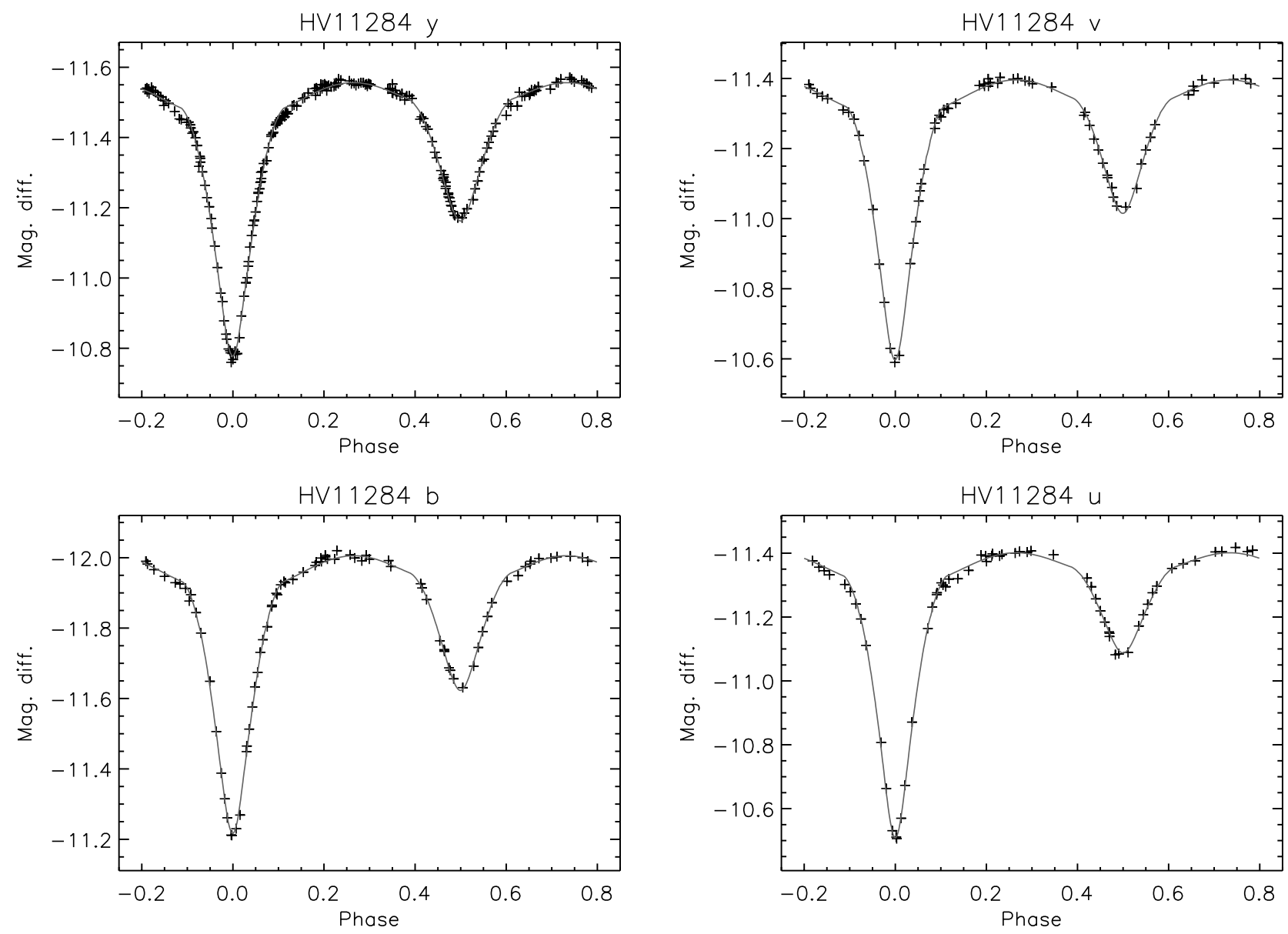

Fig. 16. uvby light curves of HV 11284 (SMC). The theoretical curves correspond to the solutions given in Table 24 .

\subsection{Ephemeris}

Two times of primary minimum and one of secondary minimum, derived from the uvby CCD observations, are given in Table 18 together with the epochs of the ephemeris by Payne-Gaposchkin \& Gaposchkin (1966) and Udalski et al. (1998b). Based on a reanalysis of the OGLE $I$ light curve, the latter has been adjusted by -0.0087 . We note that the light curve tabulated by Gaposhkin (1977) is shifted with primary minimum located at phase 0.25 , i.e. as if the epoch should correspond to maximum light level. From a weighted least squares fit to the times of primary eclipse and the two epoch, we derive the following linear ephemeris:

$$
\text { Min } \mathrm{I}=2449343.5996+3.6254398 \times E .
$$

The period is somewhat shorter than that determined from the photographic Harvard observations (3.625487), and somewhat longer than the result (3.62537) published by Udalski et al. (1998b). However, a reanalysis of their I (DIA; see below) light curve yields 3.62545 .

\subsection{Light curve analysis, photometric elements}

From the shape of the light curves, the two components of HV 11284 are expected to be larger and more deformed
Table 18. Times of minima for HV 11284 (LMC). Phases are calculated from the ephemeris given in Eq. (5). References are: SAO9, Payne-Gaposchkin \& Gaposchkin (1966); U1998, Udalski et al. (1998b) adjusted by -0.0087 ; C2003, this paper.

\begin{tabular}{lccrc}
\hline \hline HJD-2 400 000 & rms & Type & Phase & Reference \\
\hline 34682.321 & & $\mathrm{P}$ & 0.0000 & SAO9 \\
48975.607 & 0.005 & $\mathrm{~S}$ & 0.4971 & $\mathrm{C} 2003$ \\
49343.598 & 0.002 & $\mathrm{P}$ & -0.0004 & - \\
49633.636 & 0.002 & $\mathrm{P}$ & 0.0003 & - \\
50471.1116 & & $\mathrm{P}$ & 0.0001 & $\mathrm{U} 1998$ \\
\hline
\end{tabular}

than appropriate for the WINK model, and we have therefore applied only the Wilson-Devinney model for the photometric analysis; see Sect. 6.4 for details on the actual WD code. The standard $u v b y$ indices for the combined light of the components, listed in Table 4, correspond to an effective temperature of about $23000 \mathrm{~K}$ (Napiwotzki et al. 1992), which has then been adopted for the primary component. As for HV 1433, the actual effective temperature might be slightly higher, but tests show that the possible difference is of no importance for the light curve analysis. Linear limb darkening coefficients by Diaz-Cordoves et al. (1995) were adopted together with gravity exponents and reflection albedos of 1.0. A circular orbit and synchronized rotation of the components were assumed. 
Table 19. WD solutions for HV 11284 based on the two available OGLE $I$ light curves; see text for details. A mass ratio $q=0.80$ and $T_{\mathrm{p}}=23000 \mathrm{~K}$ has been adopted.

\begin{tabular}{lrr}
\hline \hline & $I($ DIA $)$ & $I$ \\
\hline$i\left(^{\circ}\right)$ & 84.32 & 84.66 \\
& \pm 14 & \pm 44 \\
$\Omega_{\mathrm{p}}$ & 3.6172 & 3.6325 \\
& \pm 49 & \pm 138 \\
$\Omega_{\mathrm{s}}$ & 4.1145 & 4.0562 \\
& \pm 97 & \pm 372 \\
$r_{\mathrm{p}}$ & 0.367 & 0.365 \\
$r_{\mathrm{s}}$ & 0.270 & 0.275 \\
$x_{\mathrm{p}}$ & 0.24 & 0.24 \\
$x_{\mathrm{s}}$ & 0.31 & 0.31 \\
$T_{\mathrm{s}}(\mathrm{K})$ & 15242 & 15183 \\
& \pm 49 & \pm 95 \\
$L_{\mathrm{s}} / L_{\mathrm{p}}$ & 0.295 & 0.309 \\
$\sigma(\mathrm{mag})$ & 0.0090 & 0.0171 \\
\hline
\end{tabular}

The stellar shapes depend on the mass ratio between the components, and on their rotational velocities, which are unfortunately not known since spectroscopic observations are not available. This means that we needed to explore various plausible configurations.

Besides the uvby light curves, we have also analyzed the $I$ light curve available from the OGLE WWW catalogue ${ }^{2}$. $B$ and $V$ light curves are also available, but they contain too few points for meaningful analyses. Two versions of the light curves are available: the original light curves are based on photometry derived from a modified DoPHOT package, and recently new and more accurate light curves based on difference image analyses (DIA) have been published (Żebruń et al. 2001; Żebruń et al. 2001). We have used the $I$ (DIA) light curve for the WD analyses. It contains 312 points obtained through a large number of nights. It is generally well covered, but rather few points are obtained during primary eclipse. The original $I$ light curve leads to nearly identical but less well defined WD solutions; see Table 19.

First, we have considered HV 11284 to consist of two detached components, and a set of $y$ and $I$ solutions for a range of adopted mass ratios between 0.6 and 1.4 are listed in Tables 20 and 21 . For all mass ratios, the hotter primary component is larger than the 8-9000 K cooler secondary component. Filling factors $F$ (see e.g. Mochnacki 1984) are between 0.91 and 0.97 for the primary, and between 0.84 and 0.72 for the secondary. Formally, the best solutions correspond to a mass ratio of around 1.2, which is, however, not very likely. For mass ratios above 1.0, and given the large temperature difference, the more massive component is expected to have evolved off the main sequence and to have become not only the cooler but also the larger component. This is clearly not supported by the light curve solutions. If, on the other hand, both components of HV 11284 are still within the main sequence band, current evolutionary models predict a mass ratio not larger than about 0.6 for a primary temperature of around $23000 \mathrm{~K}$ and a 8-9000 K temperature difference between the components.

\footnotetext{
${ }^{2}$ http://sirius.astrouw.edu.pl
}

Table 20. WD solutions for HV 11284 ( $y$ light curve) for adopted mass ratios between 0.6 and 1.4. A detached configuration is assumed, and $T_{\mathrm{p}}=23000 \mathrm{~K}$ has been adopted throughout.

\begin{tabular}{lrrrrr}
\hline \hline$q=m_{\mathrm{s}} / m_{\mathrm{p}}$ & 0.60 & 0.80 & 1.00 & 1.20 & 1.40 \\
\hline$i\left(^{\circ}\right)$ & 84.78 & 84.95 & 85.37 & 84.38 & 82.75 \\
& \pm 11 & \pm 10 & \pm 8 & \pm 10 & \pm 7 \\
$\Omega_{\mathrm{p}}$ & 3.3971 & 3.6418 & 3.9088 & 4.1889 & 4.4898 \\
& \pm 61 & \pm 49 & \pm 30 & \pm 39 & \pm 42 \\
$\Omega_{\mathrm{s}}$ & 3.3725 & 4.0714 & 4.8617 & 5.5874 & 5.9983 \\
& \pm 59 & \pm 101 & \pm 94 & \pm 148 & \pm 175 \\
$r_{\mathrm{p}}$ & 0.3679 & 0.3633 & 0.3557 & 0.3466 & 0.3353 \\
$r_{\mathrm{s}}$ & 0.2824 & 0.2737 & 0.2622 & 0.2588 & 0.2726 \\
$F_{\mathrm{p}}$ & 0.91 & 0.94 & 0.91 & 0.97 & 0.95 \\
$F_{\mathrm{s}}$ & 0.84 & 0.82 & 0.74 & 0.72 & 0.72 \\
$x_{\mathrm{p}}$ & 0.34 & 0.34 & 0.34 & 0.34 & 0.34 \\
$x_{\mathrm{s}}$ & 0.37 & 0.37 & 0.37 & 0.37 & 0.37 \\
$T_{\mathrm{s}}(\mathrm{K})$ & 15160 & 14790 & 14393 & 14212 & 14249 \\
& \pm 40 & \pm 37 & \pm 35 & \pm 34 & \pm 34 \\
$L_{\mathrm{s}} / L_{\mathrm{p}}$ & 0.314 & 0.286 & 0.260 & 0.259 & 0.304 \\
$\sigma(\mathrm{mag})$ & 0.0097 & 0.0086 & 0.0080 & 0.0078 & 0.0079 \\
\hline
\end{tabular}

Table 21. WD solutions for HV 11284 (OGLE I (DIA) light curve) for adopted mass ratios between 0.6 and 1.4. A detached configuration is assumed, and $T_{\mathrm{p}}=23000 \mathrm{~K}$ has been adopted throughout.

\begin{tabular}{|c|c|c|c|c|c|}
\hline$q=m_{\mathrm{s}} / m_{\mathrm{p}}$ & 0.60 & 0.80 & 1.00 & 1.20 & $\overline{1.40}$ \\
\hline \multirow[t]{2}{*}{$i\left(^{\circ}\right)$} & 83.22 & 84.32 & 84.18 & 83.29 & 81.86 \\
\hline & \pm 23 & \pm 14 & \pm 14 & \pm 9 & \pm 7 \\
\hline \multirow[t]{2}{*}{$\Omega_{\mathrm{p}}$} & 3.3861 & 3.6172 & 3.8983 & 4.1957 & 4.5005 \\
\hline & \pm 97 & \pm 51 & \pm 47 & \pm 35 & \pm 41 \\
\hline \multirow[t]{2}{*}{$\Omega_{\mathrm{s}}$} & 3.3861 & 4.1114 & 4.8585 & 5.4805 & 5.8599 \\
\hline & \pm 142 & \pm 97 & \pm 184 & \pm 128 & \pm 122 \\
\hline$r_{\mathrm{p}}$ & 0.3695 & 0.3668 & 0.3572 & 0.3457 & 0.3340 \\
\hline$r_{\mathrm{s}}$ & 0.2868 & 0.2696 & 0.2625 & 0.2651 & 0.2803 \\
\hline$x_{\mathrm{p}}$ & 0.24 & 0.24 & 0.24 & 0.24 & 0.24 \\
\hline$x_{\mathrm{s}}$ & 0.31 & 0.31 & 0.31 & 0.31 & 0.31 \\
\hline \multirow[t]{2}{*}{$T_{\mathrm{s}}(\mathrm{K})$} & 15648 & 15242 & 14999 & 14870 & 14985 \\
\hline & \pm 58 & \pm 49 & \pm 45 & \pm 43 & \pm 43 \\
\hline$L_{\mathrm{s}} / L_{\mathrm{p}}$ & 0.349 & 0.294 & 0.279 & 0.302 & 0.364 \\
\hline$\sigma(\mathrm{mag})$ & 0.0100 & 0.0090 & 0.0080 & 0.0075 & 0.0075 \\
\hline
\end{tabular}

Attempts to improve the fit of the theoretical light curves to the observations for mass ratios around 0.6 by adjusting rotational velocities, reflection coefficients, and limb darkening coefficients (including using non-linear laws) have not been very successful, and adding a small amount of third light from the possible close companion does not help much either, as seen from Table 22. Without knowing the mass ratio, it is, however, still considered too early to exclude definitively that HV 11284 is a detached system. Analyses of the $b, v$, and $u$ light curves lead to similar but less accurate results and conclusions. In Table 23 we compare the $u v b y$ solutions for an arbitrary (although not very likely) mass ratio of 0.8 which, at the moment, seem to represent the deformation of the components adequately, although not as well as higher mass ratios.

Next, we have considered HV 11284 to be a semi-detached system with the cooler component filling its Roche lobe. For this configuration, we also find it impossible to determine safely the mass ratio from the light curve solutions. It is, 
Table 22. WD solutions for HV 11284 ( $y$ light curve) for adopted mass ratios between 0.6 and 1.4. A detached configuration has been assumed, and $T_{\mathrm{p}}=23000 \mathrm{~K}$ has been adopted throughout. A small amount of 3rd light of $0.06\left(L_{3} /\left(L_{\mathrm{p}}+L_{\mathrm{s}}\right)\right)$ has been included.

\begin{tabular}{lrrrrr}
\hline \hline$q=m_{\mathrm{s}} / m_{\mathrm{p}}$ & 0.60 & 0.80 & 1.00 & 1.20 & 1.40 \\
\hline$i\left(^{\circ}\right)$ & 86.02 & 86.04 & 86.06 & 86.29 & 84.83 \\
& \pm 13 & \pm 13 & \pm 10 & \pm 8 & \pm 8 \\
$\Omega_{\mathrm{p}}$ & 3.4103 & 3.6442 & 3.9034 & 4.1715 & 4.4576 \\
& \pm 47 & \pm 45 & \pm 35 & \pm 34 & \pm 40 \\
$\Omega_{\mathrm{s}}$ & 3.2994 & 3.9514 & 4.6699 & 5.4515 & 6.0543 \\
& \pm 42 & \pm 83 & \pm 80 & \pm 96 & \pm 93 \\
$r_{\mathrm{p}}$ & 0.3659 & 0.3629 & 0.3565 & 0.3490 & 0.3394 \\
$r_{\mathrm{s}}$ & 0.2931 & 0.2859 & 0.2766 & 0.2669 & 0.2696 \\
$x_{\mathrm{p}}$ & 0.34 & 0.34 & 0.34 & 0.34 & 0.34 \\
$x_{\mathrm{s}}$ & 0.37 & 0.37 & 0.37 & 0.37 & 0.37 \\
$T_{\mathrm{s}}$ & 15280 & 14934 & 14595 & 14307 & 14175 \\
& \pm 38 & \pm 35 & \pm 33 & \pm 33 & \pm 34 \\
$L_{\mathrm{s}} / L_{\mathrm{p}}$ & 0.347 & 0.318 & 0.293 & 0.273 & 0.288 \\
$\sigma(\mathrm{mag})$ & 0.0095 & 0.0085 & 0.0077 & 0.0075 & 0.0079 \\
\hline
\end{tabular}

Table 23. WD solutions for HV 11284 for an adopted mass ratio of $q=$ 0.8 . A detached configuration has been assumed, and $T_{\mathrm{p}}=23000 \mathrm{~K}$ has been adopted throughout.

\begin{tabular}{lrrrr}
\hline \hline & $y$ & $b$ & $v$ & $u$ \\
\hline$i\left(^{\circ}\right)$ & 84.95 & 85.00 & 85.10 & 84.52 \\
& \pm 10 & \pm 20 & \pm 32 & \pm 45 \\
$\Omega_{\mathrm{p}}$ & 3.6418 & 3.6849 & 3.7161 & 3.7443 \\
& \pm 49 & \pm 76 & \pm 105 & \pm 162 \\
$\Omega_{\mathrm{s}}$ & 4.0714 & 4.1212 & 4.1270 & 3.9734 \\
& \pm 101 & \pm 164 & \pm 288 & \pm 479 \\
$r_{\mathrm{p}}$ & 0.363 & 0.357 & 0.353 & 0.349 \\
$r_{\mathrm{s}}$ & 0.274 & 0.269 & 0.268 & 0.284 \\
$x_{\mathrm{p}}$ & 0.34 & 0.38 & 0.40 & 0.40 \\
$x_{\mathrm{s}}$ & 0.37 & 0.43 & 0.46 & 0.43 \\
$T_{\mathrm{s}}(\mathrm{K})$ & 14790 & 14852 & 15310 & 14178 \\
& \pm 37 & \pm 65 & \pm 72 & \pm 79 \\
$L_{\mathrm{s}} / L_{\mathrm{p}}$ & 0.286 & 0.272 & 0.275 & 0.212 \\
$\sigma(\mathrm{mag})$ & 0.0086 & 0.0086 & 0.0084 & 0.0101 \\
\hline
\end{tabular}

however, seen that only values below $0.5-0.6$ lead to acceptable agreement between the theoretical light curves and the observations. The quality of the fits is comparable to those for detached solutions, meaning that a semi-detached configuration for HV 11284 is certainly possible. Solutions for an adopted mass ratio of $q=0.4$ are listed in Table 24 .

In conclusion, we find relative radii of $0.33-0.36$ (primary) and $0.26-0.30$ (secondary) for the components and a temperature difference of of 8-9000 K between them, but the actual configuration of HV 11284 remains an open question. The orbital inclination is $82^{\circ}-85^{\circ}$.

\subsection{Discussion}

The uvby and OGLE $I$ light curves of HV 11284 are well covered and of quite high quality. They should allow definitive photometric solutions once the mass ratio is established from spectroscopic observations. HV 11284 is potentially a valu-
Table 24. WD solutions for HV 11284 for an adopted mass ratios of $q=0.4$. A semidetached configuration with the less massive secondary component filling its Roche lobe has been assumed, and $T_{\mathrm{p}}=$ $23000 \mathrm{~K}$ has been adopted throughout.

\begin{tabular}{lrrrrr}
\hline \hline & $I$ (DIA) & $y$ & $b$ & $v$ & $u$ \\
\hline$i\left(^{\circ}\right)$ & 81.77 & 82.71 & 82.26 & 82.10 & 82.97 \\
& \pm 6 & \pm 5 & \pm 8 & \pm 8 & \pm 16 \\
$\Omega_{\mathrm{p}}$ & 3.4145 & 3.3410 & 3.4436 & 3.7161 & 3.3378 \\
& \pm 142 & \pm 81 & \pm 110 & \pm 105 & \pm 253 \\
$\Omega_{\mathrm{s}}$ & 2.6781 & 2.6781 & 2.6781 & 2.6781 & 2.6781 \\
$r_{\mathrm{p}}$ & 0.338 & 0.347 & 0.334 & 0.324 & 0.347 \\
$r_{\mathrm{s}}$ & 0.303 & 0.303 & 0.303 & 0.303 & 0.303 \\
$x_{\mathrm{p}}$ & 0.24 & 0.34 & 0.38 & 0.40 & 0.40 \\
$x_{\mathrm{s}}$ & 0.31 & 0.37 & 0.43 & 0.46 & 0.43 \\
$T_{\mathrm{s}}(\mathrm{K})$ & 16056 & 15638 & 15773 & 16175 & 15166 \\
& \pm 43 & \pm 37 & \pm 85 & \pm 61 & \pm 70 \\
$L_{\mathrm{s}} / L_{\mathrm{p}}$ & 0.506 & 0.446 & 0.462 & 0.486 & 0.305 \\
$\sigma(\mathrm{mag})$ & 0.0081 & 0.0092 & 0.0085 & 0.0086 & 0.0119 \\
\hline
\end{tabular}

able system for distance determination, and since it is relatively bright, we suggest that such observations are done, e.g. at the VLT.

\section{Concluding remarks}

With present-day facilities, precise studies of eclipsing binaries in the LMC and the SMC have become possible. Such objects are excellent distance indicators, and distance moduli better than \pm 0.10 mag can often be obtained. Also, accurate absolute dimensions for MC binary components are important for the study of internal structure, mass loss and evolution of massive metal-deficient stars.

We have in this paper, as part of a large scale project on MC eclipsing binaries, presented new complete $u v b y$ light curves and analyses of them for the LMC systems HV 982 and HV 12578, and the SMC system HV 11284, and partial results for the SMC system HV 1433. Accurate standard uvby indices have also been established for each binary, and individual interstellar reddenings have been determined.

HV 982 and HV 12578 are well-detached systems with eccentric orbits, each consisting of components of comparable sizes, and accurate photometric elements have been established. Adopting the spectroscopic elements given by Fitzpatrick et al. (2002) for HV 982, we have derived absolute dimensions of its component which agree well with their results. A distance modulus of $V_{0}-M_{V}=18.63 \pm 0.08$ is obtained, corresponding to a distance of $53.2 \pm 2.0 \mathrm{kpc}$, which is in formal agreement with (although slightly larger than) their determination. In a forthcoming paper, we will combine our photometric results with those underway from high-dispersion spectra obtained with the UVES spectrograph on the ESO VLT and present absolute dimensions, chemical abundances and distances for HV 12578 and refined results for HV 982.

HV 1433 and HV 11284 (identical to the OGLE - II system SMC_SC5_140701) both consist of two rather close, deformed and quite different stars. Because the mass ratios between the components - and their rotation rates - are not known, definitive photometric elements could not be obtained. We have, 
however, presented a sample of possible photometric solutions. Since HV 11284 is relatively bright and has complete uvbyI light curves, we recommend that spectroscopy is obtained for this SMC system. In addition to spectroscopy, more photometry is also needed for HV 1433.

Acknowledgements. This research, which is based on observations carried out at the Danish $1.5 \mathrm{~m}$ telescope at ESO, La Silla, Chile, has been supported by the Danish Natural Science Research Council through research grants to the project "Structure and evolution of stars; the distance scale of the Universe. New insight from studies of eclipsing binaries and pulsating stars", carried out at Aarhus University and Copenhagen University, and through its Centre for Ground-Based Observational Astronomy. ESO is gratefully acknowledged for granting a 2-months studentship to SSL in 1995, during which parts of the data reduction were carried out. SSL was furthermore partially supported by National Science Foundation grant number AST9900732. This research has made use of the Simbad database, operated at CDS, Strasbourg, France.

\section{References}

Alcock, C., Allsman, R. A., Alves, D., et al. 1997, AJ, 114, 326

Andersen, J., Clausen, J. V., \& Nordström, B. 1984, A\&A, 137, 281

Andersen, J., Clausen, J. V., Nordström, B., \& Reipurth, B. 1983, A\&A, 121, 271

Bell, S. A., Hill, G., Hilditch, R., et al. 1991, MNRAS, 250, 119

Bell, S. A., Hill, G., Hilditch, R. W., Clausen, J. V., \& Reynolds, A. P. 1993, MNRAS, 265, 1047

Buonnano, R., Buscema, G., Corsi, C. E., Ferraro, I., \& Iannicola, G. 1993, A\&AS, 106, 275

Clausen, J. V. 2000, in From Extrasolar Planets to Cosmology: The VLT Opening Symposium, ed. J. Bergeron, \& A. Renzini (Springer), 225

Clausen, J. V., Giménez, A., \& Scarfe, C. D. 1986, A\&A, 167, 287

Clausen, J. V., Larsen, S. S., Garcia, J. M., Giménez, A., \& Storm, J. 1997, A\&AS, 122, 559

Crawford, D. L. 1978, AJ, 83, 48

Dekker, H., D’Odorico, S., Kaufer, A., Delabre, B., \& Kotzlowski, H. 2000, in Proc. Conf. SPIE 4008-61

Diaz-Cordoves, J., Claret, A., \& Giménez, A. 1995, A\&AS, 110, 329

D'Odorico, S. 2000, The Messenger, 99, 2

Ferrarese, L., Silbermann, N. A., Mould, J. R., et al. 2000, PASP, 112, 177

Duncan, S. P. R., Tobin, W., Watson, R. D., \& Gilmore, A. C. 1993, MNRAS, 265, 189

Fitzpatrick, E. L., Ribas, I., Guinan, E. F., et al. 2002, ApJ, 564, 260

Flower, P. J. 1996, ApJ, 469, 355

Gaposhkin, S. I. 1970, Spec. Rep. 310, Smithsonian Astrophys. Obs. Gaposhkin, S. I. 1977, Spec. Rep. 380, Smithsonian Astrophys. Obs.

Gibson, B. K. 2000, Mem. Soc. Astron. Italiana, 71, 693

Giménez, A., \& Garcia-Pelayo, J. 1983, Ap\&SS, 92, 203

Giménez, A., \& Bastero, M. 1995, Ap\&SS, 226, 99

Grison, P., Beaulieu, J.-P., Pritchard, J. D., et al. 1995, A\&AS, 74, 331

Groenewegen, M. A. T., \& Salaris, M. 2001, A\&A, 366, 752

Guinan, E. F., Fitzpatrick, E. L., DeWarf, L. E., et al. 1998, ApJ, 509, L21

Hodge, P. W., \& Wright, F. W. 1967, The Large Magellanic Cloud (Washington)

Hodge, P. W., \& Wright, F. W. 1977, The Small Magellanic Cloud (Washington)

Jensen, K. S., Clausen, J. V., \& Giménez, A. 1988, A\&AS, 74, 331

Kjeldsen, H., \& Frandsen, S. 1992, PASP, 104, 413
Kreiner, J. M. 1972, Rocz. Astr. Obs. Krakowskiego, 43, 98

Kurucz, R. K. 1992, in The Stellar Population of Galaxies (Dordrecht: Reidel), ed. B. Barbuy, \& A. Renzini, Proc. IAU Symp., 149, 225

Kwee, K. K., \& van Woerden, A. 1956, BAN, 12, 327

Lafler, J., \& Kinman, T. D. 1965, ApJS, 11, 216

Larsen, S. S. 1999, A\&AS, 139, 393

Larsen, S. S. 1996, Master Thesis, Astronomical Observatory, Copenhagen University

Larsen, S. S., Clausen, J. V., \& Storm, J. 2001, A\&A, 364, 455

Mochnacki, S. W. 1984, ApJS, 55, 551

Napiwotzki, R., Schönberner, D., \& Wenske, V. 1993, A\&A, 268, 653

Nelsen, C. A., Cook, K. H., Popowski, P., \& Alves, D. R. 2000, AJ, 119,1205

Niemela, V. S., \& Bassino, L. P. 1994, ApJ, 437, 332

Nordlund, A., \& Vaz, L. P. R. 1990, A\&A, 228, 231

Ostrov, P. G., Lapasset, E., \& Morrell, N. I. 2000, A\&A, 356, 935

Paczyński, B. 1997, in The extragalactic distance scale, ed. M. Livio, M. Donahue, \& N. Panagia, STSI Symp. Ser., 10, 273 (Cambridge Univ. Press)

Payne-Gaposchkin, C. H. 1971, Smithson. Contrib. Astrophys., 13

Payne-Gaposchkin, C. H., \& Gaposhkin, S. I. 1966, Smithson. Contrib. Astrophys., 9

Pritchard, J. D., \& Niemela, V. 2000, in From Extrasolar Planets to Cosmology: The VLT Opening Symposium, ed. J. Bergeron, \& A. Renzini (Springer), 232

Pritchard, J., Tobin, W., Clark, M., \& Guinan, E. F. 1998a, MNRAS, 297, 278

Pritchard, J., Tobin, W., Clark, M., \& Guinan, E. F. 1998b, MNRAS, 299, 1087

Ribas, I., Jordi, C., \& Giménez, A. 2000a, MNRAS, 318, L55

Ribas, I., Guinan, E. F., Fitzpatrick, E. L., et al. 2000b, ApJ, 528, 692

Ribas, I., Fitzpatrick, E. L., Malony, F. P., \& Guinan, E. F. 2002, ApJ, 574,771

Salaris, M., \& Groenewegen, M. A. T. 2002, A\&A, 381, 440

Schechter, P. L., Mateo, M., \& Saha, A. 1993, PASP, 105, 1342

Shapley, H., Nail, V., \& McKibben 1942, Harv. Bull., 916, 19

Shapley, H., Nail, V., \& McKibben 1953, Proc. Nat. Acad. Sci. USA, 39,1

Stetson, P. 1987, PASP, 99, 191

Udalski, A., Pietrzynśki, G., Woźniak, P., et al. 1998a, ApJ, 509, L25

Udalski, A., Soszyński, I., Szymański, M., et al. 1998b, Acta Astron., 48, 563

Vaz, L. P. R. 1984, Ph.D. Thesis, Copenhagen University, unpublished

Vaz, L. P. R. 1986, Rev. Mex. Astron. Astrof., 12, 177

Vaz, L. P. R., Andersen, J., \& Rebello Soares, M. C. A. 1995, A\&A, 301, 693

Vaz, L. P. R., \& Nordlund, Å. 1985, A\&A, 147, 281

Watson, R. D., West, S. R. D., Tobin, W., \& Gilmore, A. C. 1992, MNRAS, 258, 527

Welch, D. L., \& Stetson, P. B. 1993, AJ, 105, 1813

West, S. R. D., Tobin, W., \& Gilmore, A. C. 1992, MNRAS, 254, 419

Westerlund, B. E. 1990, A\&AR, 2, 29

Westerlund, B. E. 1997, The Magellanic Clouds (Cambridge University Press)

Wilson, R. E. 1994, PASP, 106, 921

Wilson, R. E., \& Devinney, E. J. 1971, ApJ, 166, 605

Wood, D. B. 1971, AJ, 76, 701

Żebruń, K., Soszyński, I., \& Woźniak, P. R. 2001, Acta Astron., 51, 303

Żebruń, K., Soszyński, I., Woźniak, P. R., et al. 2001, Acta Astron., 51,317 